\title{
Key Technologies and Development Trends in Advanced Intelligent Sawing Equipments
}

Yangyu Wang ${ }^{1,2,3}$, Yongle Zhang ${ }^{1}$, Dapeng Tan ${ }^{1,2,3^{*}}$ and Yongchao Zhang ${ }^{4}$

\begin{abstract}
As a starting point in equipment manufacturing, sawing plays an important role in industrial production. Intelligent manufacturing equipment is an important carrier of intelligent manufacturing technologies. Due to the backwardness of intelligent technology, the comprehensive performance of sawing equipments in China is obviously different from that in foreign countries. State of the art of advanced sawing equipments is investigated along with the technical bottleneck of sawing machine tool manufacturing, and a new industrial scheme of replacing turning-milling by sawing is described. The key technologies of processing-measuring integrated control, multi-body dynamic optimization, the collaborative sawing network framework, the distributed cloud sawing platform, and the self-adapting service method are analyzed; with consideration of the problems of poor processing control stableness, low single machine intelligence level, no on-line processing data service and active flutter suppression of sawing with widewidth and heavy-load working conditions. Suggested directions for further research, industry implementation, and industry-research collaboration are provided.
\end{abstract}

Keywords: Sawing equipments, Processing-measuring integrated control, Collaborative sawing framework, Intelligent network middleware, Sawing cloud service platform

\section{Introduction}

\subsection{Sawing Equipments}

As one of the eleven categories of metal cutting machine tools, sawing equipment play an important role in industrial production, driving output valued at more than 200 billion yuan. With the continual emergence of new energy, space, and marine resource utilization and the development of other related fields utilizing major, large-scale equipments, such as million-kilowatt nuclear power equipments, giant ships, large aircraft, etc., the working conditions of the core components of industrial production are increasingly extreme. Most applications use large-sized, hard-cutting metal materials with excellent mechanical properties, and their production and manufacturing are increasingly dependent on the abilities of heavy-duty manufacturing equipments with

\footnotetext{
${ }^{*}$ Correspondence: tandapeng@zjut.edu.cn

${ }^{1}$ College of Mechanical Engineering, Zhejiang University of Technology, Hangzhou 310023, China

Full list of author information is available at the end of the article
}

the capability of dealing with large-scale applications $[1,2]$. Heavy manufacturing equipment is a basic and important key component in the production chain of the manufacturing industry, which reflect the extreme manufacturing capacity and manufacturing level of the country [3-5]. It is an important guarantee for national defense security and national economic industrial security. As an example of large-scale intensive manufacturing, we take the large-sized shaft and cylinder parts of nuclear power equipments shown in Figure 1.

They are obtained by cutting the forging blank as shown in Figure 2, using heavy-duty sawing machine to cutting large-scale components (Figure 3 ). The width of the cutting slot is only about $3 \mathrm{~mm}$. The process has obvious advantages, such as lower raw material consumption, protection of the environmental, and the absence of a heat-affected zone. With continuous improvement of machining accuracy and an increase in production efficiency requirements, especially in strategic engineering fields such as aerospace, nuclear power, the chemical industry, shipping, rail transit, etc., which have brought 


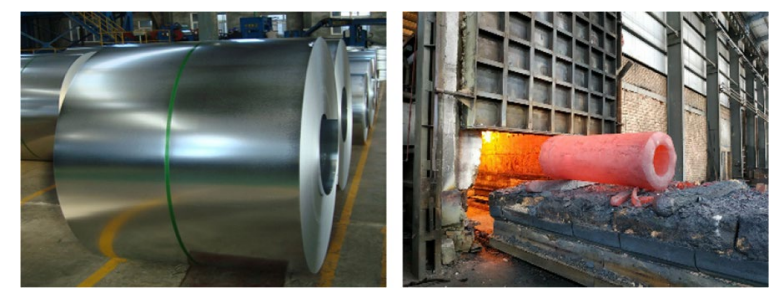

Figure 1 Large parts in nuclear power equipment
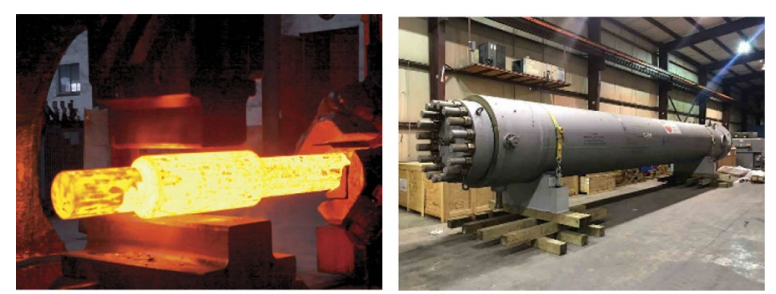

Figure 2 Forgings of the tube connecting section and shaft parts
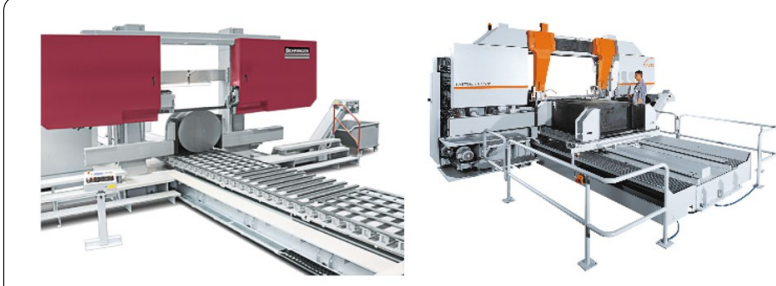

a Round bar

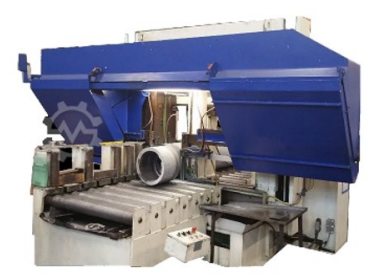

c Seamless steel tube

b Square steel

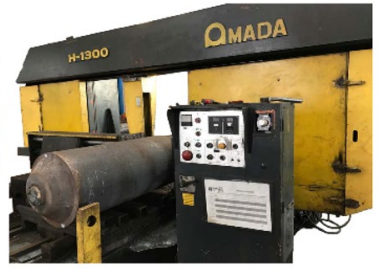

d Shaft

Figure 3 Wide range and heavy load cutting of heavy-duty sawing machine

important challenges to sawing equipment performance $[6,7]$.

Difficult-to-machine materials have poor thermal conductivity, severe work-hardening, high specific strength, and large unit cutting force. They are prone to produce chatter and to emit a sharp whine in heavy load sawing, resulting in a sharp decline in sawing efficiency, tool life, and surface quality [8]. In China, the automatic monitoring and intelligent control technology of the sawing status have become a challenging problem in the development and manufacture of advanced sawing equipments. In particular, a long-term foreign monopoly has seriously affected China's industrial security in advanced sawing equipment technologies. Therefore, the research of advance sawing equipments in order to facilitate replacing turning-milling by sawing has broad engineering application prospects.

To enhance the capabilities created by innovation and international competitiveness, and seize a commanding lead in the competitive field, China has proposed to implement five major projects in the Made in China 2025 plan [9]. In it, advanced equipments innovation projects are listed. By 2025, China aims to achieve a substantial increase in its market share of advanced equipments with independent intellectual property rights, significantly reduce its dependence on core technologies from foreign countries, and step up to an internationally leading level of equipments in important fields. General Secretary Xi Jinping has pointed out that the pillars of a great power must be in its own hands, and we must rely on our own efforts to force improvement of independent innovation abilities [10-12]. Over past years, this author team has conducted thorough research on common technical bottlenecks and key requirements of the sawing equipment industry. It has aimed to help break through the technical bottleneck and the foreign technology monopoly, helping China's advanced sawing equipment manufacturing industry improve quality and efficiency, and serve important engineering fields such as aerospace, shipbuilding, nuclear power, high-speed rail, automobile production, etc.

\subsection{Bottleneck Problem of Intelligent Sawing Equipments} In the past ten years, China's level of production level of sawing equipments have been significantly improved. However, due to a lack of original innovation, most enterprises still focus on the production of medium and low-grade machine tools [13]. The sawing equipment industry structure is comprised of vicious competition in the low-grade product area, disorder in the intermediate products field, and a complete ineffectiveness in the sophisticated product sector. To better meet the users' requirements for high speed, composite, intelligent and environmental protection of sawing machines, foreign manufacturing enterprises of sawing machine have deeply integrated machine design, Internet and information technology, and artificial intelligence technology. Sawing process gradually presents the trend of network and intelligence. Then, the intelligent technology of sawing machine has been further developed, and it is increasingly obvious that the gap between domestic and foreign sawing machine in their various performance indexes, as shown in Table 1. Key technologies, such as the cutting database, automatic servo feed control system, and 


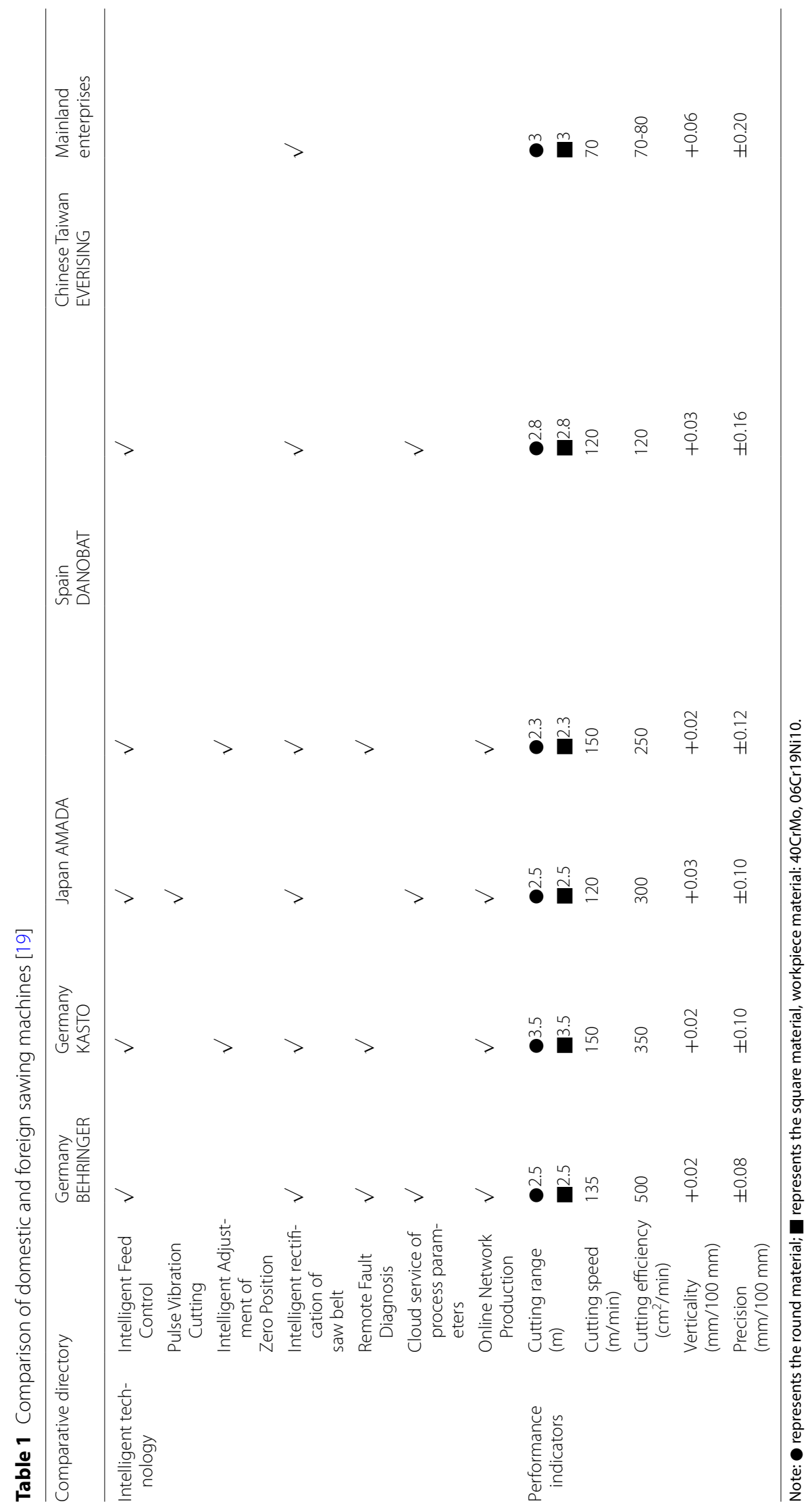


multi fluid cooling system enable foreign band sawing machines, whose production is headed by German company BEHRINGER, to realize high-efficiency sawing of difficult-to-cut materials with high hardness, high strength, and high density. Taking HBM440A-SC and HBM540A-SC double column band sawing machines as examples, productivity tests show that the BEHRINGER sawing machine has a cutting efficiency of $500 \mathrm{~cm}^{2} / \mathrm{min}$ when cutting $40 \mathrm{CrMn}$ high-strength alloy steel, while the domestic sawing speed can only reach $77 \mathrm{~cm}^{2} / \mathrm{min}$ [14-16]. The PCSAW series sawing machine from Japanese company Amada has adopted Automatic Feed Control (AFC) technology which can adjust sawing speed and feed pressure in real time according to the material, shape, and the time interval of the tool in and out. With original dual pulse vibration cutting technology, the vibration noise caused by the feed and the main cutting motion can be reduced, thereby realize the doubling of sawing efficiency and cutting precision $[17,18]$. Some well-known foreign enterprises such as the German company KASTO, the American company DOALL, and the Spanish company DANOBAT [19-22] are dedicated to research on sawing technology for difficult-to-machine materials. Their products have characteristics and advantages in structural design, manufacturing process, noise reduction, and vibration absorption.

In general, there is a large gap between China's upscale sawing equipments and advanced-level foreign equipments in terms of machine tool structure stiffness, cutting tools, $\mathrm{CNC}$ systems, and intelligent management and control services [23-25]. There exist the following bottleneck problems:

(1) Poor processing control stableness, prone to produce resonance howling.

In China, the sawing machine industry was founded later, and there are fewer research results and technical data on key technologies, such as structural optimization design, sawing mechanisms, and control strategies [26-28]. At present, domestic enterprises rely mainly on referencing foreign advanced sawing equipments and experience to design the mechanical structures of sawing machines, which lack intelligent control of the process [29-31]. The Kasto-respond system, developed by KASTO (a sawing machine manufacturer of Germany), can intelligently identify changes in the cutting contact length of thick-walled and thin-walled materials, even hard spots. And then the operator can change the processing feed rate in real time to maintain constant cutting force.

(2) Low single machine intelligent level-carries out large-scale collaborative production with difficulty.

At present, advanced sawing equipments abroad have realized the universal applications of intelligent sawing and formed an integrated process. The process includes computer-aided management of production operations and of the material consumption budget, the computer-aided design of parts, the automatic nesting of cutting parts, and the direct generation of an NC code for processing. This makes sawing more stable, faster, and more efficient [32, 33]. AMADA, a sawing machine manufacturer of Japan, establishes a cloud cutting-parameter database based on more than 20 years of sawing experience. By connecting the machine tools to the Internet, the operator can select the appropriate cutting parameters intelligently only by inputting the size and material of the cutting workpiece. The domestic traditional sawing machine emphasizes single machine automation, which can realize the functions of automatic feeding, automatic counting, automatic detection of materials, and the detection of tape breakage. However, due to the lack of a flexible design of the control system, the feed speed of the sawing cannot be automatically adjusted in the face of different processing materials, and so it is difficult to achieve constant power sawing, intelligent saw band correction, the intelligent selection of sawing parameters, intelligent shape recognition, intelligent fault diagnosis, remote wireless monitoring, real-time intelligent feedback control, and other functions [34-36].

(3) A lack of online sawing data services-difficulty in locking in the optimal control targets for machining-the machine tool operates inefficiently.

At present, domestic sawing equipments do not have the functionality of on-line work, and usually adopts a working mode of off-line control planning and single machine independent operation [37-39]. The workshop development of the intelligent factory is distributed through on-line discrete control, implementing remote monitoring, fault diagnosis, material scheduling, etc. Therefore, the relevant equipments must be based on fully controlling the continuous operation production line control system of the sawing machine, which the current domestic saw machine control system cannot achieve. BEHRINGER, a sawing machine manufacturer of Germany, provides independently developed intelligent control system for each sawing machine. On the one hand, users can program freely according to the working conditions, on the other hand, remote fault diagnosis and networked production can be realized. And they can fully consider customer needs and improve production efficiency. Due to differences in processing materials, workpiece shapes, and cutting tools, controlling changes in sawing force is difficult and forces the sawing machine to reduce the feed speed in the sawing process of large-scale and difficult to machine materials. Thus, the working efficiency of the sawing machine decreases. 


\subsection{New Formats of Cutting Processing-"A New Industrial} Scheme of Replacing Turning-Milling By Sawing "

As an important link in mechanical manufacturing, compared with other traditional cutting methods (Turning, Milling, etc.), metal sawing has the characteristics of good material adaptability, high production efficiency and no thermal shadow zone. Due to the poor surface quality of processing (the research of surface quality mainly focuses on the surface roughness), sawing is usually the first process of metal processing. After that, users need to use other processes to meet the surface quality requirements of the workpiece, such as rough turning, semi-rough turning, finish turning, etc. [40, 41].

After long-term development, the types of metal sawing machines are gradually increasing in order to meet the needs of metal primary processing and machinery manufacturing and other related industries. And it mainly includes the following three types: circular sawing machine, band sawing machine and hacksaw machine. Among them, hacksaw machines are being gradually eliminated due to their low cutting efficiency. Because of the left and right eccentric teeth design of the band saw blade, the surface of the workpiece will appear "washboard" trace. And the surface of the workpiece to be as rough as $50 \mu \mathrm{m}$. Compared with band saws, circular saws have higher cutting precision, and the surface roughness of the workpiece is usually around $25 \mu \mathrm{m}$. The machining accuracy of rough turning is IT12-IT11, and the surface roughness is $50-12.5 \mu \mathrm{m}[42,43]$. Therefore, we can use metal circular sawing to replace rough turning in some working conditions with low surface roughness. In the process of machining, reducing the working procedure can reduce the workpiece position change and improve the machining accuracy. Moreover, it can improve the production efficiency of the workpiece, reduce the cost and enhance the market competitiveness. At present, some foreign sawing enterprises rely on the accumulation of years of technological innovation and the application of intelligent technology to process the surface accuracy of workpiece, which can reach the rough milling level, and some models can even reach the semi-rough milling level. Taking Germany BEHRINGER Company as an example, the company has successfully developed a sawing-drilling-milling machine tool(VP-2X 350-12D BS) for a high performance drilling-milling compound production line. The multi-purpose machine with hardstamping, high speed drilling and milling and sawing. Therefore, it can greatly improve the processing efficiency, reduce time and processing cost for user enterprises, which increase the use and reliability of sawing equipment in the market.

Under the background of intelligent manufacturing, it is a powerful tool to promote the upgrading of traditional manufacturing industry. And the production process become automation, intelligence, precision and green, which is an important support for the cultivation and development of machine tool industry [44, 45]. Obviously, "replacing turning-milling by sawing" has become the development trend of high-end intelligent sawing equipment in China. Overcoming the above bottleneck technology, include processing-measuring integrated control, multi-body dynamic optimization, the collaborative sawing network framework, the distributed cloud sawing platform, and the self-adapting service, which can realize the full localization of advanced sawing equipments. Meanwhile it is of great significance for the formation of a new business form of "replacing turningmilling by sawing", and for improving the service capacity of sawing equipment industry for aerospace, nuclear power, high-speed rail way and other strategic fields.

\section{Key Technologies of Advanced and Intelligent Sawing Equipments}

\subsection{Processing-Measuring Integrated Control System}

Processing-measurement integration refers to the organic integration of measurement technology and processing technology, i.e., measuring the model of the workpiece, analyzing and modifying the measured data by computer, and automatically selecting optimal cutting parameters to machine the workpiece with high precision and a complex shape [46-48]. In considering the explicit/implicit relationship of multi-dimensional conditional constraints in the precision machining process, Jia et al. [49] proposed a compensation mechanism for critical geometric dimension errors and established an online machiningmeasurement integration mechanism association model. They initially proposed a strategy of processing-measurement integrated control for forming complicated surfaces. Guan et al. systematically described the main technical challenges concerning the integrated system of sensing-transmission-control in the complex industrial network environment. They analyzed the difficult technologies involved in a complicated industrial network, such as distributed sensing, adaptive transmission, collaborative control of the process, and embedded equipment configuration and dynamic excitation response [50]. Ding et al. analyzed error sources in integrated measurement-operation-machining technology for precision/ultra-precision machining. The measured point cloud data were used to generate a robotic machining path, which then a force controlled robot can use to realize precision machining [51]. Generally, the working principle of the measure-machining integration technology as follow: by constructing the information link between the machine tool and the workpiece processing technology, the machine control system can obtain the 
geometric and physical information and carry out analysis, decision-making and control. Base on this, we can reduce the production cost of complex surface machining with high performance and improve the machining accuracy of workpiece. But in complex industrial networks, many difficulties need to be addressed. On the one hand, it is impossible to establish accurate physical models for control with traditional methods, on the other hand, a large number of operating data reflecting the machining process are generated at all times. Therefore, based on practical constraints, cost reduction and other factors, it also requires great technical problems that how to use these massive data to meet the increasing system reliability requirements. Nascimento et al. [52] proposed an alternative method based on data-driven soft sensors working through vibration measurements. Through the method, vibration signals can be measured online and closed-loop feedback gathered during the assembly and testing of refrigeration compressors - thus improving the level of control for the production process. Fountas et al. [53] proposed intelligent 3D machining trajectory planning based on swarm-based evolutionary algorithms. Through their method, optimized 3-axis sculptured surface $\mathrm{CNC}$ machining can be used in the milling process. Strbac et al. [54] researched the significance of particular factors (temperature drift, systematic error, asymmetric error) in coordinate measuring machine (CMM) measurements. They proposed a design of experiments (DoE) method for measurement uncertainty in ultra-precision machining. By applying the method, precise measurements for rising high temperature can be achieved through the processing contact. Scicluna et al. [55] presented low-speed control in the process of precision machining using a real-time commissioning method. The method can be used for low/zero speed control for permanent magnet synchronous machines (PMSMs).

Tan et al. [56-58] developed an embedded system of embedded multi-loop parallel processing-measuring integrated controls focusing on the characteristics of high intensity and high impact for sawing as shown in Figure 4. It can effectively restrain the harmonic content of the driving voltage by the linkage control of the loops associated with the sawing process. Thus, the sawing machine becomes more stable under high frequency impact conditions, and the cutting speed becomes faster. The system includes the controller, software, and the basic components of the control function. The multi-dimensional piezoelectric sensor is designed independently according to the characteristics of the sawing process. It can be measured with an intelligent sensor, such as the normal radial component and transverse component of the cutting force and the moment. These six components can be calibrated through an adaptive regression algorithm.
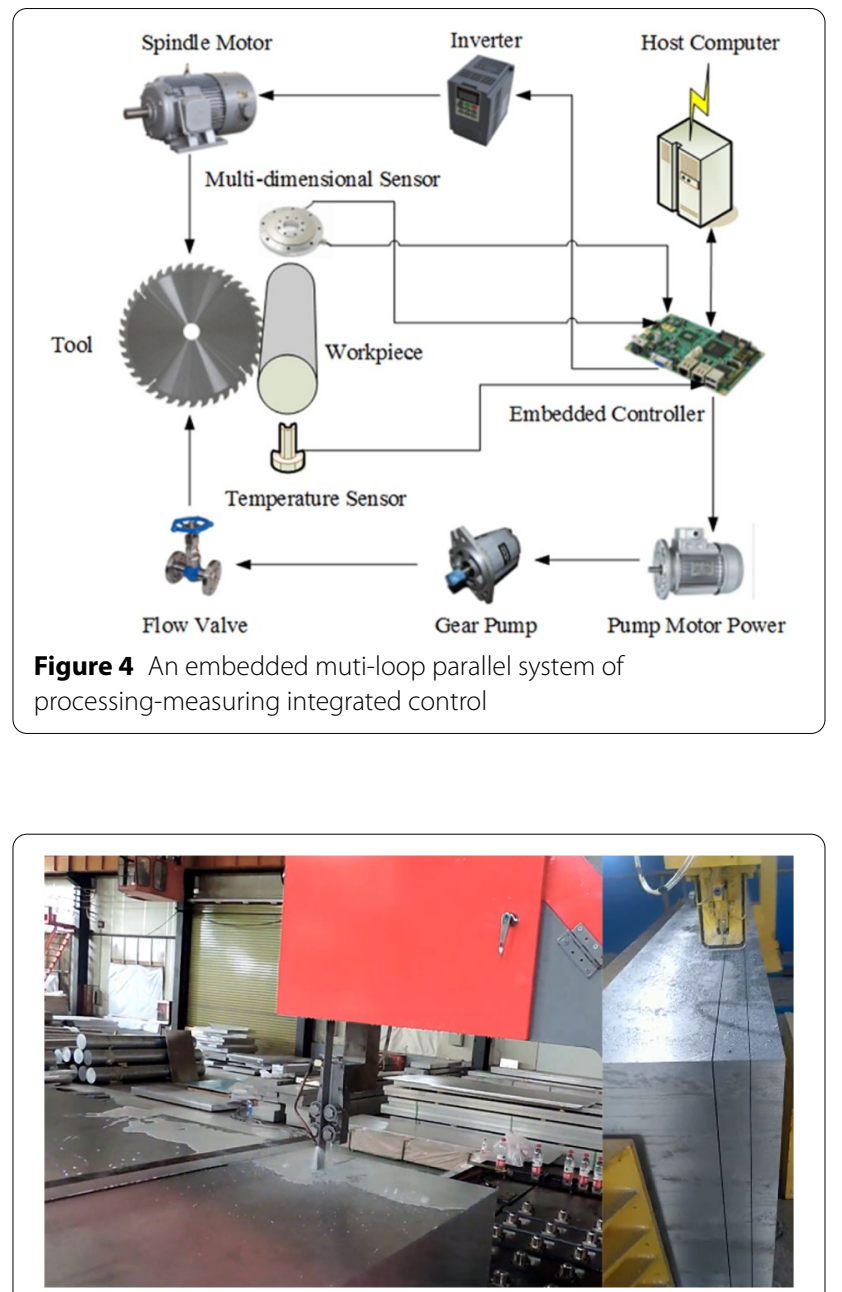

Figure 5 Cutting super sized aviation aluminium alloy

Based on this, the performance of sawing equipments can be greatly increased using technologies such as pulse cutting, remote monitoring and maintenance, intelligent saw band correction, and intelligent shape recognition. Taking the advanced sawing machine as the carrier, the system can seamlessly connect with the manufacturing execution system (MES). In addition, it has the intelligence functions of saw band correction, selection of sawing parameters, shape recognition, fault diagnosis, remote wireless monitoring, real-time feedback control, etc. The system has been successfully applied to a sawing machine for the cutting of aviation aluminum material with a large stroke, as shown in Figure 5. Its efficiency can reach more than $2700 \mathrm{~cm}^{2} /$ min when the machining stroke is $5000 \mathrm{~mm}[59,60]$.

\subsection{Intelligent Multi-Body Dynamic Optimization}

Advanced sawing equipments raise requirements for the dynamic characteristics of key and support parts because 
of its high stability and high machining accuracy. At present, such equipments have attracted increasing attention from researchers for the design and optimization of the multi-body or key parts. It is the necessary precondition to obtain vibration characteristic under the excitation of the machining load by establishing a multi-body dynamic model, according to the structure and working mode of the sawing machine.

The gearbox is an important component of a highspeed sawing machine. Its vibration performance directly affects the dynamic characteristics of the sawing machine, which in turn affects the sawing accuracy and service life of the saw blade. Related studies [52, 61, 62] on this process suggest that $90 \%-95 \%$ of radiated sound energy is transmitted along this pathway. Therefore, the minimization of vibrations and the gearbox noise under working conditions is a critical unresolved problem. $\mathrm{Gu}$ et al. [63] used topology optimization to optimize the target structure and built a new structure model based on the original one. The purpose of topology optimization is to find the shortest transmission path of the target structure and then we can greatly reduce the cost of the structure. However, in the optimization process, it is also faced with the problem of structural re-analysis due to the continuous iteration of solving controls and feasible domains. Jiang et al. [64] proposed several robust optimization design methods. Compared to traditional optimization schemes, robust optimization design improves the performance of a system at the expense of mass and maximum vibration amplitude. Li et al. [65] introduced a physical programming method to the robust design method and focused on optimizing gearbox design according to demand, based on sensitivity analysis. Sensitivity method is widely used in structural design, whereas the traditional sensitivity still has some limitations in practical application. When the design variable is within the constraint range, the sensitivity method can reflect the actual physical condition. However, when the design variable reaches its constrained boundaries, the traditional sensitivity method cannot accurately reflect the actual physical condition. Wang et al. [66] introduced the food chain conduction response algorithm for gearbox optimization and improved the comprehensive optimization performance of the algorithm based on the transmission mechanism of the middle layer of the food chain. Liu et al. [67] proposed a hybrid, user-defined element method (HUELM) to analyze the coupling dynamic characteristics of the gear transmission process. They developed the dynamic vibration model and obtained modal parameters of the gear transmission system under different working conditions. Belingardi et al. [68] proposed a multi-body dynamic modeling and analysis method for gearbox power transfer, which can obtain the function of dynamic frequency response and transmission error of the system. Sensitivity analysis refers to the degree to which a change of structural design parameters affects the objective performance function. Moreover, it quantifies the influence of a parameter change on the change of the objective function [69-71]. In the process of sensitivity analysis optimization, the designer selects effective design parameters quickly and purposefully according to the actual needs in order to improve the optimization efficiency [72-74]. Based on the complexity of the structure of the saw machine gearbox, the multi-objective function can be solved by combining the Kriging model with the MOGA algorithm, and the response surface model of the design variables and the objective function of the gearbox can be determined.

$\mathrm{Ni}$ et al. [75] established a numerical model for the sawing gearbox using numerical simulation software and quantified the problems involved in the structure optimization process. The geometrical dimensions and material parameters were initially set according to the specifications of the manufacturing process of the gearbox. As shown in Figure 6, stress concentration can easily occur for the holes and parts near the model boundary, and local refinement was carried out. The contact position between the gearbox and slider is imposed as a completely fixed constraint, which limits the model base to six degrees of freedom in the $X, Y$, and $Z$ directions and satisfies the initial structural optimization requirements. Considering the significant reduction in the computational burden due to the meshing of various parts in the assembly, the conservation of computing resources, and accuracy of the solution, the calculation model is solved by adding point mass. In Figure 7, the mass and centroid coordinates of the transmission shaft (including bearings, gears and other components) are determined by the physical model of the gearbox. The mass and centroid coordinates are added to the gearbox as point masses.

Based on the above research, the multi-body dynamic model is established through a modeling method with

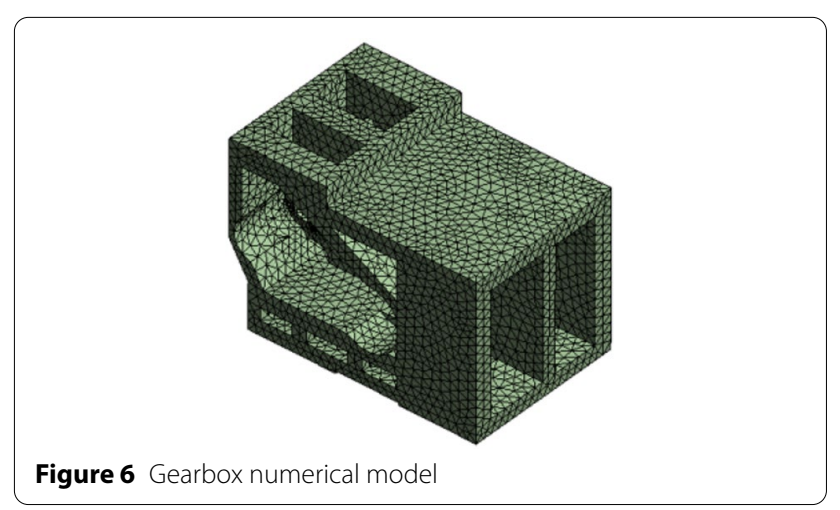




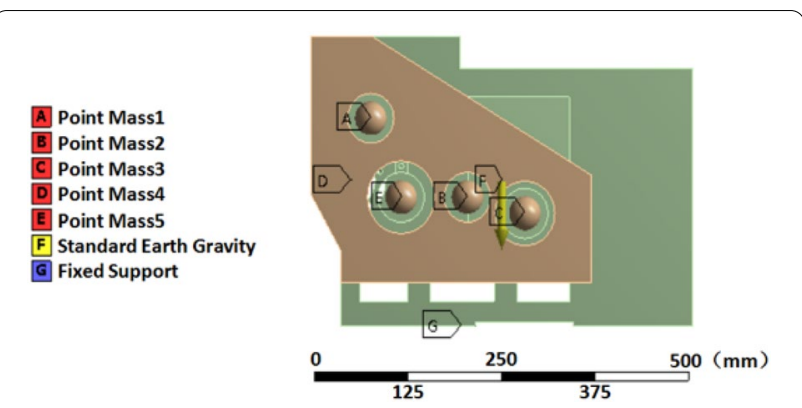

Figure 7 Mass point distribution of gearbox

mass points and receives the vibration characteristics under the excitation of the load in the sawing process [76-79]. The sensitivity of different design variables of the new gearbox was determined via a sensitivity analysis of the objective function. The optimal solution is obtained by an optimization method of the multi-objective genetic algorithm (MOGA). Then, combined with the operational modal analysis (OMA), the actual vibration characteristics of the gearbox under different working conditions are obtained, and the saw gearbox structure is optimized. Figure 8 shows the vibration signal waterfall

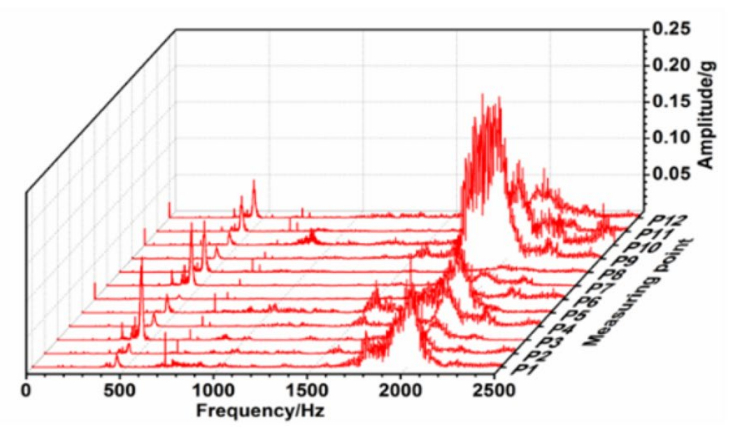

a

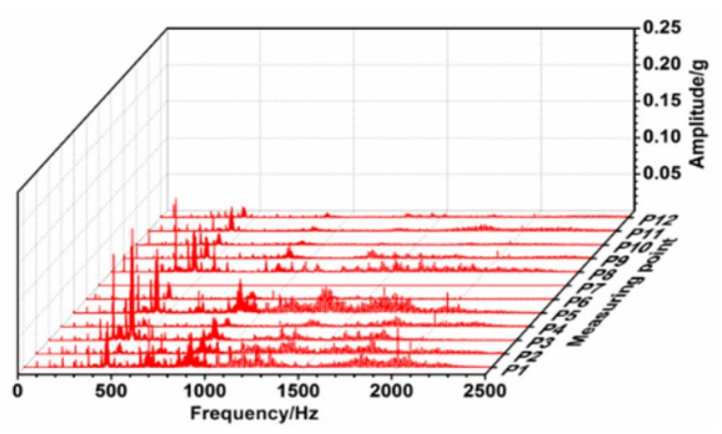

b

Figure 8 Gearbox vibration signal waterfall map (70 r/min): a before optimization, $\mathbf{b}$ after optimization diagram at $70 \mathrm{r} / \mathrm{min}$ before and after improvement of the gearbox. The improvement of the high-frequency band and low-frequency band is evident. Figure 9 shows the 3D surface topography of the workpiece sawing end face under $70 \mathrm{r} / \mathrm{min}$ conditions. It is evident from the figure that the cutting quality of the optimized circular sawing machine was improved compared to pre-optimization, whereas the surface corrugations before optimization are haphazard with irregular deep pits in some areas. This phenomenon may be due to insufficient rigidity of the circular sawing machine, which leads to flutter during the sawing process.

\subsection{Intelligent Plug And Play Sawing Network Architecture} Behind the integration of manufacturing and the Internet we see not just the transformation of the production organization, but also a fundamental change from production to sales under a new round of activity in scientific and technological innovation. Digitization, networking, and the intelligent transformation of production is becoming the core of enterprises' competitiveness in the future [80-82]. Equipment-level interconnection and

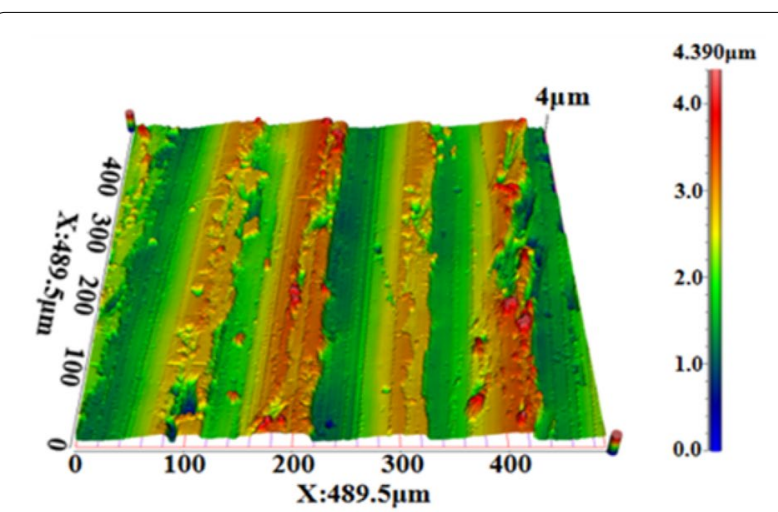

a

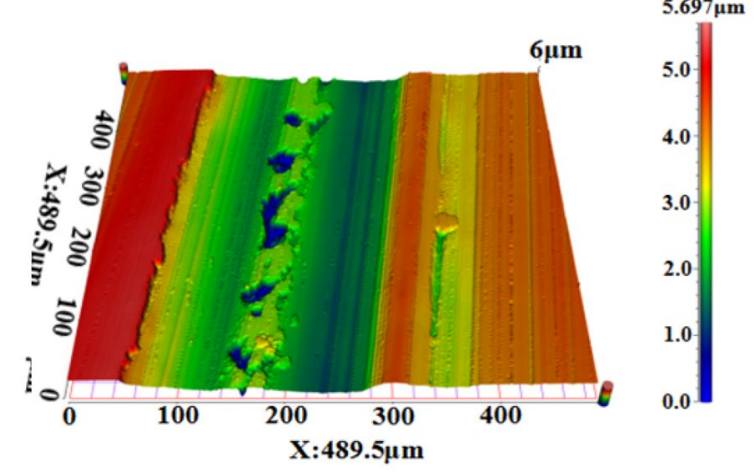

b

Figure 9 3D surface topography before and after optimization (90 r/ $\mathrm{min})$ : $\mathbf{a}$ before optimization, $\mathbf{b}$ after optimization 
collaborative manufacturing is an effective way to reduce positioning errors in systems and improve the efficiency of complex production tasks. As intelligent network middleware, Universal Plug and Play (UPnP) extends plug and play. It can effectively solve the protocol barrier of specificity in industrial networks and the problems of automatic identification caused by various kinds of equipments, so that different devices and networks can be seamlessly linked [83-85]. It provides a reliable technical underpinning for the realization of the goals set out above [86, 87]. Fan et al. proposed the use of middleware to ensure consistency of data interaction in the workflow application in an operating environment based on a unified platform of information resources. They presented a mapping method for data consistency from the business model to middleware, and from middleware to the workflow model based on eXtensible Markup Language (XML) [88]. Under networked manufacturing mode, enterprises can make full use of various manufacturing resources, such as structured data (machining database, tool library) and application tools (CAD, CAM, CAE). Based on it, manufacturing resources can be fully shared, which ultimately contribution to improve product quality and reduce costs. However, there are some problems in the networked production of traditional manufacturing system network technology, such as poor coordination and lack of dynamic configuration support. It has become a very important research problem that how to quickly locate manufacturing resources to meet the requirements of enterprises and achieve plug and play of the resources. Pan et al. proposed an intelligent system architecture of the manufacturing network based on UPnP and established a model of a network system for manufacturing automation. They used an UPnP internal communication interface to design the network communication protocol and service invocation mechanism and achieved intelligent management of a manufacturing network system [89, 90]. Zhang, Xu et al. [91-93] proposed a service architecture for device networks based on DPWS for the intelligent monitoring of distributed micro-grid devices. The service architecture combined collaborative Petri nets (CPN) technology to realize the collaborationexclusion control of devices with microgrid nodes. Tan et al. [94] introduced intelligent network middleware into the field of distributed intelligent manufacturing and proposed a pervasive computing service method for networked manufacturing monitoring. It can realize adaptive control of the digital manufacturing unit, mutual aid service, and big data support for remote streaming media. And the system can perform self-discovery, zeroconfiguration, smooth links and other adaptive functions. However, there are still some problems to be studied, such as network composite allocation, system stability optimization, and so on. Hao [27] proposed a service method of time-aware target reconstruction for the problem of multi-time-constrained production service promotion in distributed network manufacturing. It can achieve an online promotion recommendation of strategies for production services. Nee et al. [95] systematically elaborated the application research of pervasive computing methods in advanced manufacturing. They pointed out that dynamic responses of network topology architecture, intelligent control of network behavior, real-time processing of message mapping, and the mutual service of production information are the key elements in the design of middleware systems oriented at the manufacturing process. China Mobile has released 5G industrial module products, based on Haisi 5G module middleware. They can reduce the difficulty of the development of the 5G terminal, shorten the development cycle, and meet the needs of diversified industries; and effectively promote the commercial application of 5G technology in rich spectrum of scenarios including intelligent manufacturing [96-98].

Tan et al. $[99,100]$ introduced intelligent network middleware into the sawing processing area. They combined the control technology of embedded systems to build a complex network model of distributed sawing based on UPnP, as shown in Figure 10.

Based on the above-mentioned complex network model of distributed sawing process monitoring, and combined with the principle of model evolution, Tan et al. studied the propagation law of information load under the conditions of complex links. They proposed a real-time optimization and evaluation method for communication protocol and a service mechanism based on UPnP. They established a general monitoring standard, communication protocol, and interface mode for the sawing process, and created functions of automatic

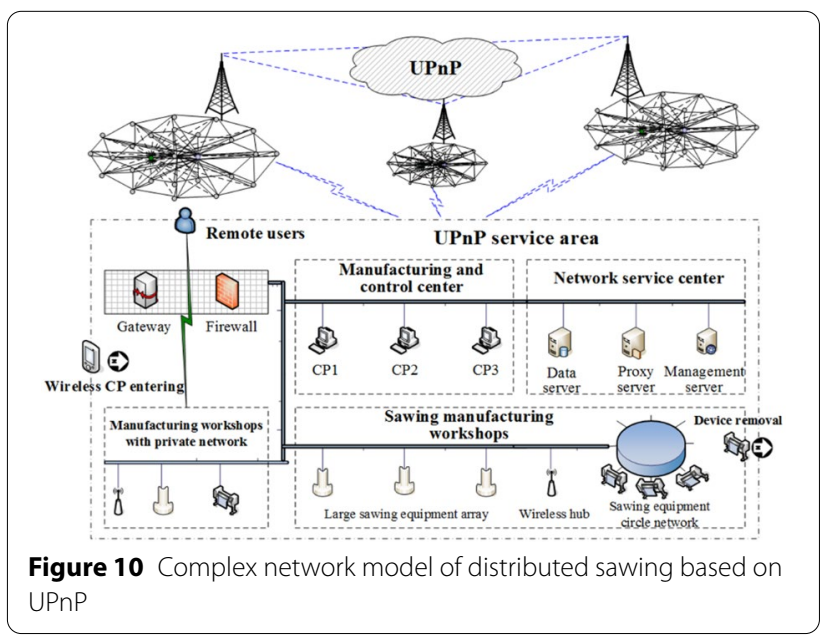


recognition, zero configuration, and seamless links of each digital sawing unit. An adaptive and intelligent plug and play digital saw cutting unit was built (see Figure 11). It improved the level of single intelligent and multimachine collaborative work.

The above service methods can provide effective adaptive ability and high response speed for changes of the network structure and enhance information exchange ability between components of sawing equipments. The efficiency and flexibility of distributed digital sawing systems are improved, most significantly the efficiency of collaborative production. In this way, digitally driven and intelligent production of sawing can be realized, and a theoretical basis and technical foundation for cloud manufacturing in sawing can be provided.

\subsection{Distributed Cloud Service Platform of Sawing Production And Self-Adapting Service Methods}

Establishing a support platform of manufacturing servitization to support the transformation of manufacturing enterprises from single product suppliers to overall

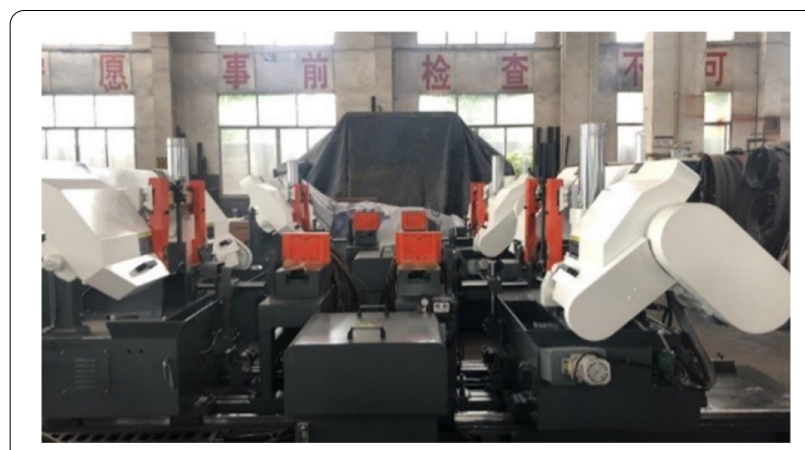

Figure 11 A plug-and-play digital sawing unit solution providers, and to provide online monitoring, remote diagnosis, maintenance, and other services are important development trends in the manufacturing industry $[34,38,101,102]$. The current boom in mobile computing and online cloud services offers a potential path to this goal, as shown in Figure 12.

$\mathrm{Li}$ et al. proposed a new intelligent manufacturing system in the era of "intelligence + ", the Cloud Manufacturing System 3.0. They defined Cloud Manufacturing 3.0 and its system architectural-technical framework and key technologies [103]. Wang et al. [1] proposed a visual component sorting method based on Convolutional Neural Networks (CNN) for the cloud computing of a flexible manufacturing system. It can realize high efficiency, high precision classification, and ordering of the workpiece under a complex production mode. Shan et al. [104] built a cloud architecture for a service-oriented manufacturing network based on the cloud computing environment with workshops, enterprises, and customers as the main body. Through scenario analysis, the uncertainty of customer demand was described as a box set and a robust optimization method for the service-oriented manufacturing network process was proposed. Cloud manufacturing is a new model for networked manufacturing. Its concept of sharing and service can realize the transformation of enterprise knowledge resources, which from traditional classified storage and retrieval to knowledge service. Then, the manufacturing resources can be centralized controlled and efficiently allocated, the knowledge resources can be fully shared, knowledge control capabilities can be enhanced. And finally the enterprise can reduce information costs. In the meanwhile, users are not only satisfied with the passive choice of

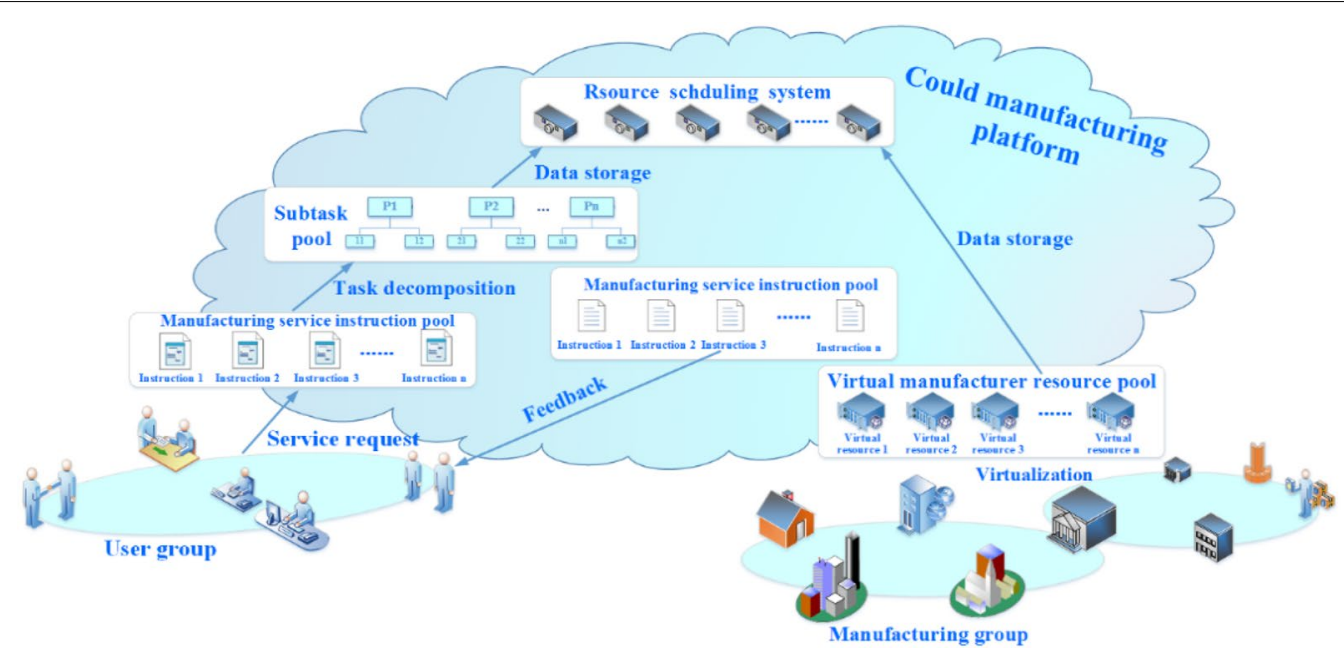

Figure 12 Cloud manufacturing architecture and online data services 
products, personalized customization has become the mainstream of manufacturing. Based on this, it must be established that the multi-time series production service mode for cloud manufacturing enterprise group. Then the enterprise can maintain competitive advantage in fierce market competition and meet customized demand of customers. Facing the production and processing of differentiated products, the single manufacturing enterprise is not the only choice. It needs more consideration that how to exchange information through cloud manufacturing service platform for the cooperative manufacturing group of differentiated products. And how to select a scientific, reasonable and feasible cloud manufacturing production process under the constraints of product service time, cost and quality. Then produce customer accredited products, optimizing service value chain under cloud manufacturing mode. Man et al. merged a Directed Acyclic Graph (DAG) on a cloud server and edge server and divided tasks based on a strategy of critical path segmentation. Their approach improves the accuracy of the distribution and realizes dynamic load balancing [105]. Helo et al. [106] proposed a cloud service method for metal processing data. They regarded data scheduling as the core service and combined it with a genetic algorithm to realize online optimization perception and recognition of production processing data. Simeone et al. [107] established a cloud platform for the optimization of the metal cutting process data form and proposed an intelligent estimation and optimization method that achieves the function and the efficiency enhancement processing of geometry form data. Mourad [108] proposed an interoperability estimation method for cloud manufacturing. By analyzing the interoperability of a manufacturing scenario context, they determined the key parameters of cloud manufacturing and gave the corresponding optimization and regulation methods of resource competition and task coherence. Refs. [35, 109-111] proposed a service platform of cloud manufacturing based on the block chain, in view of the suddenness and randomness of manufacturing information service in Industry 4.0. It enables the optimization of composite information services for dynamic production tasks. Because UPnP is not limited by a real IP address, it can easily access the internal workshop network through the external Internet and is able to transmit and process large amounts of data (such as audio data). A database service based on UPnP can be used as an independent node of the intelligent sawing production system. It is located in the data center of the whole system and undertakes tasks, such as data storage, query, management, playback, identification, and comparison. Based on the above assumptions, Tan et al.

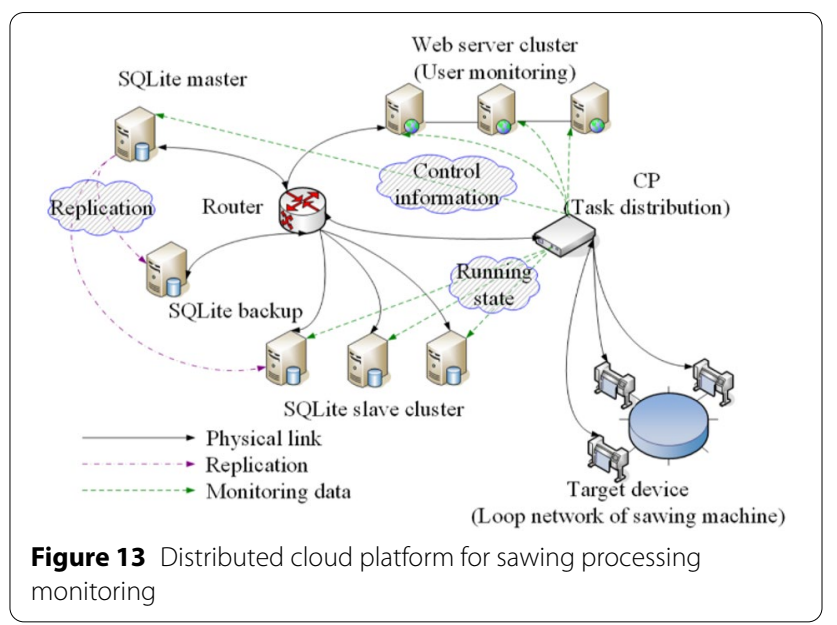

proposed a distributed cloud platform for sawing monitoring, as shown in Figure 13.

The distributed cloud platform of sawing processing monitoring is chiefly composed of the following embedded components: (1) the data collection module: adopting an algorithm combining monitoring and timing scheduling, which sets the capacity of the link list of acquisition equipment and the cycle length of the timer according to the scale of the fault diagnosis networkand so has good adaptability; (2) the network middleware module of data acquisition: an embedded UPnP Controlend-it mainly monitors and records the service information of the UPnP fault collection equipment on the network and periodically visits the working UPnP fault collection equipment in the network to obtain the operation data of the monitored machinery and save it to the database; (3) the network middleware module of database query: the embedded UPnP device mainly provides database information to the user monitoring device, prequeries according to the query conditions (such as time and device number) proposed by the user, and feeds back relevant data information to the user monitoring terminal (such as "the number of packets meeting the condition", etc.). In addition, the remote monitoring terminal is equipped with a corresponding data management and analysis system to enable real-time data display and other functions. With the support of database services, the data from any period can be selected according to specific demands for playback, data comparison, printing of reports, and other operations. At present, the database management platform of the distributed sawing monitoring network based on UPnP has completed a significant number of industrial experiments in Zhejiang Chendiao Machinery Co., Ltd., Zhejiang Rengong Machinery Co., Ltd. and in other enterprises, with stable operation and quick query. A friendly GUI has been developed by 
using the Qt/Embedded tool library, which has a Chinese environment, takes up less space, responds quickly, and switches smoothly $[112,113]$.

The online data service quality is directly determined by the network model of the distributed intelligent sawing and scheduling strategy. The key technology for the industrialization of a sawing monitoring cloud platform is to establish a multi loop-a dynamic scheduling model of a peer-to-peer device network for the service network of distributed intelligent sawing and optimize its scheduling algorithm. The mathematical model of the service network of distributed sawing is shown in Figure 14. Referring to the above model, a distributed network snifftiming scheduling $\left(\mathrm{DNS}^{2}\right)$ algorithm is proposed, which combines online node action sniffing with timing scheduling to guarantee real-time performance in accordance with basic requirements of industry monitoring [73]. In addition, the algorithm is encapsulated into a unified UPnP system event, which can adjust the capacity of the DP list and the length of the timing period according to different industrial environments. Based on the sniff mode $\left(E S^{4} \mathrm{M}\right)$ of even static-scatter-network scheduling,

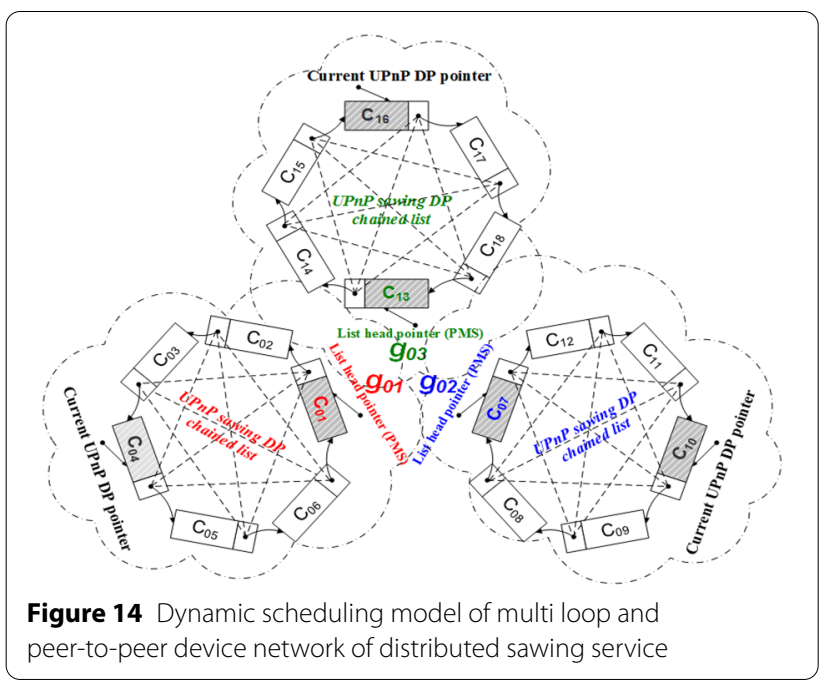

comparison shows that with an increment of the subnetwork number, the theoretical power consumption increases gradually. Under lower bandwidth, the DNS ${ }^{2}$ algorithm does not give an adequate advantage for power efficiency. The differences in power consumption of the two methods tend to be clearer when based on the increment of bandwidth. Under the conditions of maximum subnetwork number and bandwidth, the $\mathrm{DNS}^{2}$ algorithm only requires $67.5 \%$ of the power consumption of the $\mathrm{ES}^{4} \mathrm{M}$ method. Therefore, the DNS ${ }^{2}$ algorithm can reach better throughput and time delay than the $E S^{4} \mathrm{M}$ method. Moreover, the $\mathrm{DNS}^{2}$ algorithm can improve the power consumption efficiency, i.e., under the same conditions, it can transfer more data packets, increase the utilization ratio of the data link, and reduce the network idle time.

In order to verify the performance of the structure, industrial field monitoring experiments were carried out regarding common low-speed and slow-change vibration signals and high-speed and fast-change vibration signals during the sawing process (Figure 15). The working performance comparative experiment with the MySQL and Berkeley DB shows that the embedded cloud database service (EDBCS) is more effective than MySQL and the Berkeley DB in facets of real-time performance, selfadapting service, intelligent monitoring, and transaction control; especially for the embedded mobile data services involved in distributed industry monitoring. The link waiting time can decrease $75 \%$ and $58 \%$, respectively, the average READ-WRITE error ratio can decrease to less than $0.01 \%$, and the system memory space can be limited to $250 \mathrm{kB}$ (Table 2).

\subsection{On Active Flutter Suppression of Sawing with Wide-width and Heavy-load Working Conditions} Cutting chatter is a very complex mechanical vibration phenomenon in metal cutting process, which affects the processing quality of parts and limiting productivity. Heavy-load sawing has many disadvantages, such as large cutting amount, harsh cutting environment, difficulty in

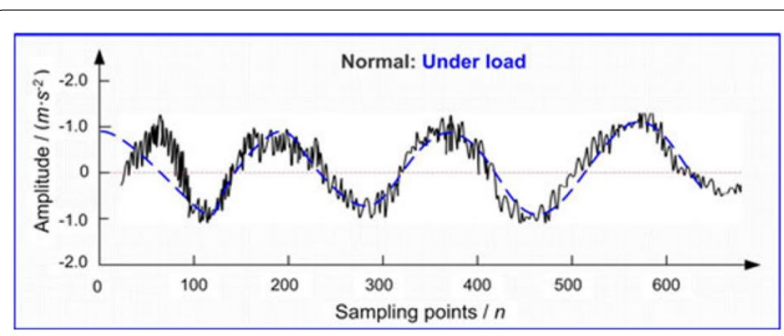

a

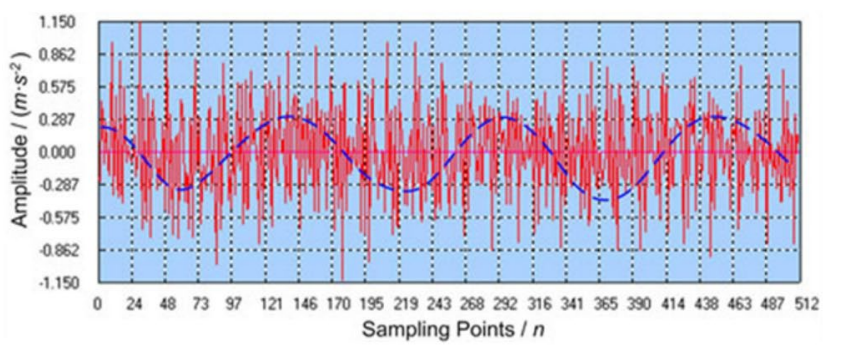

b

Figure 15 Vibration wave of spindle motor of high speed circular saw: a ECDBS standard wave, $\mathbf{b}$ real-time wave from industrial field 
Table 2 Comparative experiment of system basic technical indexes

\begin{tabular}{llll}
\hline Item & MySQL & Berkeley & ECDBS \\
\hline Self-network for DB & $\mathrm{N}$ & $\mathrm{N}$ & $\mathrm{Y}$ \\
$\begin{array}{l}\text { Industrial field online data playback and } \\
\text { comparison analysis }\end{array}$ & $\mathrm{N}$ & $\mathrm{N}$ & $\mathrm{Y}$ \\
$\begin{array}{l}\text { Intelligent transaction processing } \\
\text { Multigroup real-time scheduling }\end{array}$ & $\mathrm{N}$ & $\mathrm{N}$ & $\mathrm{Y}$ \\
$\begin{array}{l}\text { Dynamical buffer allocation } \\
\text { Max link request number }\end{array}$ & $\mathrm{N}$ & $\mathrm{N}$ & $\mathrm{Y}$ \\
Max thread table number & 50 & 120 & $\mathrm{Y}$ \\
Compatible platforms & 64 & 64 & 512 \\
Max thread buffer size & 2 & 6 & 8 \\
Mini link waiting time & $2 \mathrm{MB}$ & $4 \mathrm{MB}$ & $6 \mathrm{MB}$ \\
READ-WRITE error ratio per 100000 & $200 \mathrm{~s}$ & $120 \mathrm{~s}$ & $50 \mathrm{~s}$ \\
$\quad$ records & $0.2 \%$ & $0.3 \%$ & $<0.01 \%$ \\
Memory space & $900 \mathrm{kB}$ & $300 \mathrm{kB}$ & $<250 \mathrm{kB}$ \\
\hline
\end{tabular}

chip flow diversion and breaking, resulting in large cutting force in the cutting process. Due to the low rigidity characteristics of the trailing arm of cantilever and saw blade of the sawing structure, it is easy to produce cutting chatter after modal coupling with the process system. And severe chatter will cause scrapped parts or damage to the machine spindle. Even slight chatter can greatly reduce the cutting accuracy and blade life. Throughout the research status at home and abroad, passive flutter suppression and active flutter suppression are two major academic systems for improving the stability of cutting system. Wang et al. [114] designed a damping tool attached by $\mathrm{Mn}-\mathrm{Cu}$ alloy plates on its upper and lower surface. In this way, they improved the performance of turning $1 \mathrm{Cr} 18 \mathrm{Ni9Ti}$ stainless steel. Yang et al. [115] proposed a milling cutter structure with built-in passive damper, which length-to-diameter ratio close to 8 . And it improved the performance in cutting aluminum and titanium alloys. Ema et al. [116] applied the principle of impact damping to the design of vibration absorption boring bar. It consumes energy through the collision between the free mass block and the interior wall of the vibration damping chamber. Choudhury et al. [117] designed an active vibration reducing tool bar, which uses closed-loop control algorithm to control the piezoelectric actuator to exert reverse force on the tool bar to suppress chatter. Chiou et al. [118] used electrostatic and piezoelectric actuators to control spindle bearings. And it changed the modal characteristics of the structure to improve processing stability. Kong [119] proposed a design idea of MR intelligent boring bar with self-chattersuppression. The dynamic characteristic parameters of boring bar are changed to suppress the chatter effectively

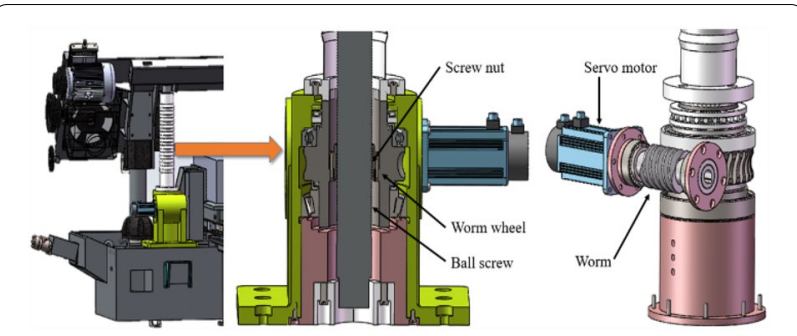

Figure 16 Servo feed device suitable for sawing machine

by adjusting the magnetic field intensity on magnetorheological material.

The primary task of passive flutter suppression is to accurately predict the stability lobe diagram. Then, the process parameters could be adjusted or other auxiliary ways, which can improve stability limit of flutter. In the sawing process, it will cause many changes under the coupling effect of the long-span simply-supported beam characteristics of the saw blade and the thin-wall characteristics, and the change of the size of the workpiece section. Such as the increment of the number of teeth involved in sawing and the cutting force. And the stability prediction has also become more difficult. Therefore, it is necessary to develop active suppression techniques based on accurate flutter state identification.

It is a common method for active flutter suppression with adjusting cutting parameters. Some high-end CNC machine tools are equipped with special control modules. And the cutting parameters such as feed rate, cutting depth and spindle speed can be adjusted in real time according to the cutting force, cutting vibration data feedback. Also, it is changed that the modal stiffness and damping of machine-tool-workpiece sawing system and the position of cutting working point on stability limit diagram. Then the cutting working point moves from the unstable region to the stable region, which finally achieve the effect of actively suppressing chatter and improving cutting stability. At present, we mainly study the flutter suppression effect of variable speed feed for sawing equipment. And in order to ensure the accuracy and reliability of the feed adjustment of sawing equipment, a servo feed device suitable for sawing machine is designed, as shown in Figure 16.

Based on this variable speed servo feed device, we combine BP neural network and fuzzy control method to study active flutter suppression control strategy in widewidth and heavy-load sawing process, as shown in Figure 17. We got tens of thousands of cutting data through our previous research. Then, the artificial neural network model is trained to establish the corresponding relationship between machining parameters and sawing force. We designed a fuzzy controller. Taking the sawing force 


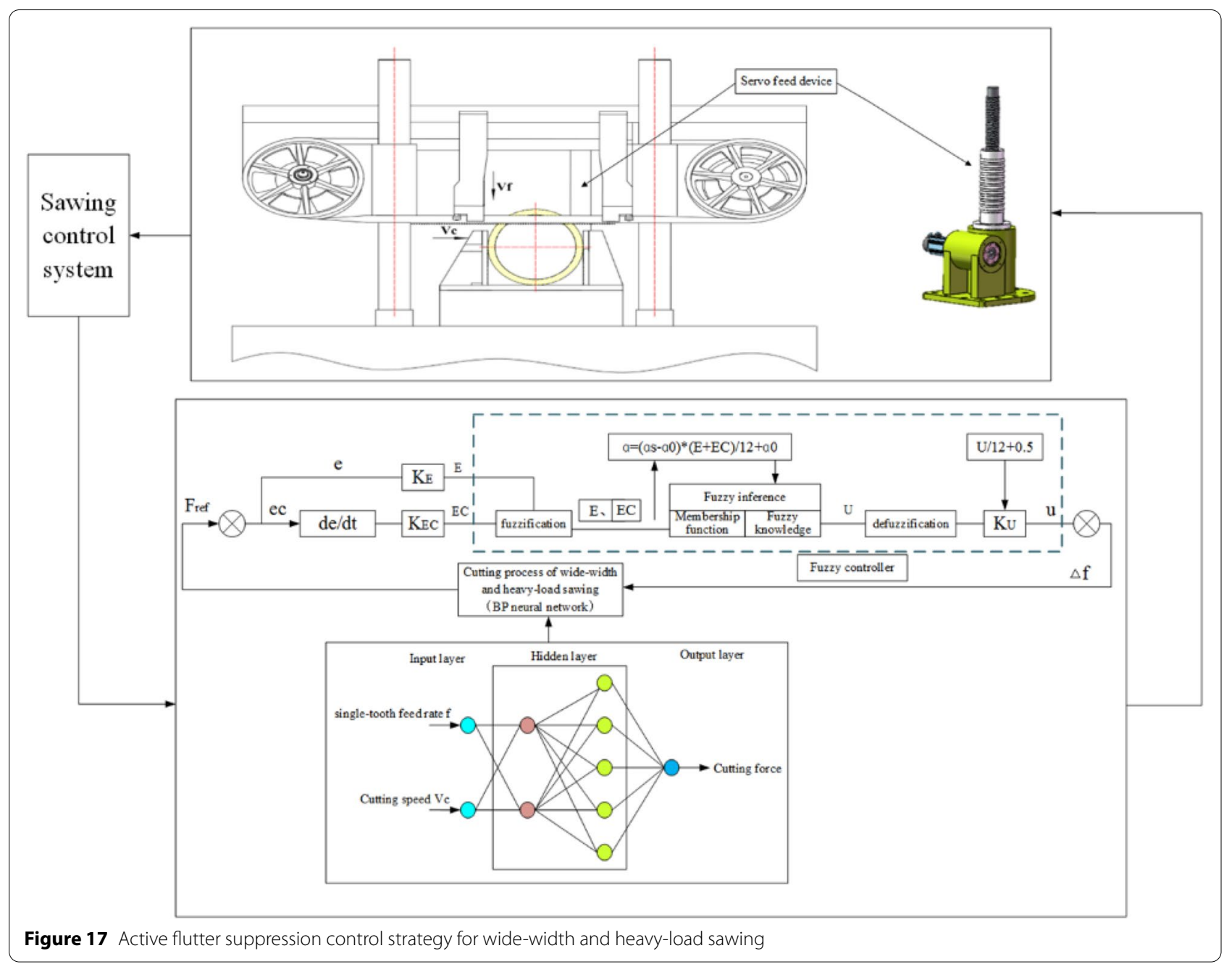

output by BP neural network as a reference, the deviation change rate is obtained by comparing with the actual cutting force, which is used as the input variable of the fuzzy controller. And the output variable is the variation of feed per tooth $\Delta f$. By finding the fuzzy relationship between the variation of feed per tooth $\Delta f$ and the deviation change rate, we can use the fuzzy control rules to constantly modify $\Delta f$. And the controlled object has excellent dynamic performance to achieve constant force control and stable sawing, and active flutter suppression of sawing.

\section{Analysis of the Current Situation and Development Trends}

\subsection{Current Situation Analysis of the Key Technologies of Intelligent Sawing}

We analyze the key technologies of processing-measuring integrated control, multi-body dynamic optimization, the collaborative sawing network framework, distributed sawing cloud platform, and self-adapting service method, while considering the problems of poor processing control stability, the low level of single machine intelligence, and no on-line processing data service. The outcomes of the analysis are as follows.

(1) The processing-measuring integrated control system is one of the research hotspots in the advanced intelligent sawing equipments manufacturing field. The self-sensing of key physical quantities and the calibration of physical signals in a high-frequency impact environment are prerequisites for realizing the integrated control of sawing processing and measurement. The key difficulty is the self-sensing technology of key physical quantities in the machining process. Current research mainly focuses on online error compensation, perceptual transmission control, adaptive trajectory optimization, speed synchronization control, etc. Processing-measurement integrated control system supporting adaptive network characteristics and intelligent self-sensing 
technologies still have much room for improvement.

(2) The main drive gearbox is the key factor affecting the overall dynamic performance of sawing equipments. The basic design idea is to facilitate a lightweight design with significant stiffness while improving design efficiency. In addition, when an optimization algorithm is used to obtain a solution, many working conditions are often ignored, resulting in a deviation between the final optimization result and actual performance. In order to optimize the rigidity and dynamic mechanical properties of sawing equipments and improve its processing quality and stability, an optimization design method that combines topology optimization, sensitivity analysis, and response surface optimization can be considered.

(3) Intelligent network middleware technology can effectively improve the vertical control depth and work flexibility of current production modes centered on MES. The large capacity access capability and communication bandwidth of $5 \mathrm{G}$ networks can provide important technical support for middleware service computing in equipment-level interconnected complex network environments. Current research on these fields focuses mainly on workflow consistency mapping, equipment network service architecture, pervasive computing manufacturing monitoring services, the optimization of production service strategies, etc. Research on intelligent middleware technology supporting equipment-level interconnected complex network scenarios and 5G access is still in its infancy. Therefore, combining intelligent network middleware with an intelligent sawing field and 5G communication technology to improve the single equipment intelligence level and collaborative production capacity can provide strong support for sawing equipments in undertaking large-scale and complex production tasks.

(4) Cloud computing has become a key supporting technology for online production data optimization services, and its importance is becoming more and more significant in the networked and large data environment. Current research focuses mainly on cloud manufacturing system architecture, the online processing of complex production tasks, scenario analysis of the manufacturing process, task path partition strategies, the online optimization of process data, etc., and these mainly focus on the level of Software as a Service (SaaS). There are few studies reporting on Infrastructure as a Service (IaaS) and Platform as a Service (PaaS) for intelligent manufacturing. Therefore, in view of the demand for online intelligent data services for sawing, we establish an embedded cloud platform for the distributed monitoring of sawing and propose a multi-loop, peer-to-peer device network dynamic scheduling algorithm-an important solution for improving real-time communication and the work flexibility of online intelligent data services for sawing.

\subsection{Development Trends of Intelligent Sawing Equipments} As a starting point in equipment manufacturing, sawing involves many process parameters and derivative data from processing objects and shows obvious randomness and disorder. Sawing processing as a typical high-speed cold processing method, has several advantages in raw material consumption, energy consumption, environmental pressure, production efficiency, the heat-affected zone, etc. First, compared with traditional turning, chipping, milling, and flame cutting, the material consumption of sawing can be reduced by $70 \%$. Second, compared with laser, flame, plasma, wire cutting, and other thermal cutting methods, the equivalent heat consumption of sawing is only $20 \%-30 \%$ of the above methods. Further, sawing has no heat-affected zone, has high safety performance, and low operation and maintenance costs. Third, the sawing equipment developed based on this project has the same working precision and material consumption as laser and wire cutting, but has obvious advantages in dimension and efficiency of processing objects. The rapid development of intelligent manufacturing technology, cloud computing, 5G communications, and other digital technologies provide important support for the design of advanced sawing equipment control systems and the on-line service of sawing processing.

At present, advanced sawing equipment technologies are developing in the direction of high-speed, high-precision, high-point manufacturing, green manufacturing, functional composites, networking, and intelligent processes. Developing intelligent sawing equipments and improving the intelligence level of the sawing equipment industry has become a consistent choice for advanced sawing equipment manufacturing enterprises. In order to step into the ranks of advanced manufacturing countries and ensure the realization of the goal of increasing manufacturing power, China's advanced sawing equipments are developing steadily in four directions as follows.

(1) Characteristic optimization design of equipments and key components.

Little research has been done on machining errors caused by the vibrations of sawing, and the capacity of research in related industries is weak. The engineering applicability of high-performance sawing intelligent 
control systems and embedded cloud data service methods under special working conditions such as special structural workpieces, difficult to process materials, high temperatures, and high humidity environments will be limited, which also presents a barrier to next steps in this field. Due to the requirements of high stability and machining accuracy of advanced sawing equipments, higher demands are being put forward for dynamic characteristics of supporting parts and key components. While ensuring lightweight design, complete machine performance, such as dynamic and static stiffness, thermal stability, seismic resistance, working efficiency, and work stability are greatly improved.

(2) Further development of digital manufacturing and intelligent control technology.

For sawing sub-fields (hard-to-cut materials, titanium alloys, non-ferrous metals, carbon fibers), it is necessary to build a highly perfected online service platform for sawing cloud data, rationally allocate production resources, and develop functional modules, such as status monitoring, fault diagnosis, forecast and warning, process optimization, and quality control, so as to improve industrial production efficiency. Creating an intelligent sawing plant with agile service requires close collaboration and on-demand reconstruction and maximized sharing of production and information resources. In the sawing process, the factory collects and transmits data in real time. The platform carries out analyses and decisions based on collected environmental parameters and processing data, so as to achieve maximum intelligence of the whole industrial process as far as possible. Intelligent manufacturing is developing rapidly, and the competition in the global manufacturing industry led by intelligent manufacturing is intensifying. Guided by the spirit of the "Integration of Artificial Intelligence and Physical Economy", the digital manufacturing mode of advanced sawing equipments has matured continuously. Equipment manufacturing enterprises have carried out deep digital transformations, explored intelligent solutions, and gradually moved to the top of value chain.

(3) The significance and practice of integrated manufacturing are deepened and extended.

Sawing and milling compound equipments and the new industrial trend of replacing turning-milling by sawing promote further development of sawing equipments towards higher performance and higher efficiency. It is necessary to construct an innovative ecology for intelligent sawing control, coordinate with domestic sawing enterprises and their users to share processing data, promote the establishment of sawing technology databases, and improve the training of service decision algorithms. In actual working conditions, the sawing process is affected by a multi-physical field, multi-scale, and other factors. At present, most enterprise modeling only considers the single physical field, but little modeling considers the influence of the flow field, temperature field, sound field, and other factors. In enterprise processing, relevant adjustment work is only carried out for fixed-point machines, and the above factors have little effect on the processing accuracy of enterprises. However, cloud data service faces different production environments for distributed manufacturing enterprises, and so it is necessary to consider the theoretical modeling of multi-physical fields.

(4) Promoting the construction and improvement of sawing cloud services.

Big data is the basis of cloud data services. In current sawing processing systems, the amount of data that can be used for training calculations is small, and the data acquisition capabilities of enterprises are insufficient, which brings obstacles to precision sawing based on cloud data services. For sawing subdivisions (hard-toprocess materials, titanium alloys, non-ferrous metals, carbon fibers), in view of special environmental conditions such as high temperature and humidity, it is key to improve the online service platform for processing cloud data of advanced sawing equipments, rationalize the distribution of production data, and thus significantly improve industrial production efficiency. Besides, due to the simple encryption mechanism of the industrial sensor itself, the industrial Internet requires tighter technological security protection. To address the above this project will improve the encryption mechanism of the service platform, distribute and store enterprise core data, and coordinate the new manmachine relationship under cloud service processing.

\section{Conclusions}

With the continuous improvement of machining accuracy and the increase in production efficiency requirements, especially in strategic engineering fields, such as aerospace, nuclear power, and high-speed rail, the performance of sawing equipments faces great technical challenges. In view of the above, the development mode of China's advanced sawing equipment industry should gradually change from introducing and absorbing to independent and original innovation. Therefore, with a focus on major national strategic needs, and considering the problems of core technology, key components, process and equipments, while also exploring collaborative innovation in the advanced sawing equipment industry chain and constructing common technologies for a collaborative innovation system of advanced sawing equipments, we can focus on the following aspects. 
(1) Advanced sawing equipments innovation and research should be dedicated to realizing multifunctional integrated control, such as intelligent sawing belt deviation correction, intelligent cutting parameter selection, intelligent shape recognition, intelligent fault diagnosis, remote wireless monitoring, real-time intelligent feedback control, etc. in order to carry out large-scale collaborative production.

(2) By means of dynamic optimization, automatic detection and intelligent control technology of sawing equipments, large-scale sawing equipments can be utilized so that sawing equipments can replace turning-milling by sawing, thus improving the control stability of processing and reducing resonance squeal.

(3) In order to integrate a large industrial database of sawing enterprises, collect industrial supply chain data, enterprise production and sales data, equipments operation data, a joint processing center of advanced sawing equipments should be constructed to provide online sawing data service, control the continuous sawing machine production line, accurately target processing optimization control, and improve the efficiency of machine tool operation.

(4) There should be a focus on developing an integrated control system of embedded sawing and cutting processing-measuring, building a framework of intelligent sawing and cutting collaborative production networks, building a distributed sawing and cutting production cloud service platform of "industry sharing, enterprise specific pecial", advancing the embedded adaptive service method, and realize realizing the information integration control of complex surface digital processing.

(5) Finally, big data analysis should be used to guide industry development and providing enterprises with data analysis such as product quality benchmarks, comparison analysis, sales market analysis, technology research, and developmental direction to solve common technical problems and promote industry development.

In addition, industrial enterprises and university scientific research institutes should establish a long-term cooperation mechanism of production, learning, and research; form a new distributed and networked scientific research institute cluster; provide technical support for sawing equipment manufacturing enterprises-especially for small and medium-sized enterprises; fill the gap between basic research in universities and research in scientific research institutes, and provide product and industrial technology innovation with enterprises. Such steps promise to advance effective and sustainable development of advanced sawing equipments in China.

\section{Acknowledgements}

Not applicable.

\section{Authors' contributions}

YW determined the overall framework and central idea of the article; YZ was in charge of the literature search and wrote part of the manuscript; $Y Z$ wrote part of the manuscript; DT checked and approved the manuscript. All authors read and approved the final manuscript.

\section{Authors' information \\ Yangyu Wang, born in 1979, is currently an associate professor at Zhejiang University of Technology, China. He received his PhD degree from Zhejiang University of Technology, China, in 2013. His research interests include the processing technology and equipments for cutting, mechanical structure and dynamics.}

Yongle Zhang, born in 1996, is currently a master graduate at College of Mechanical Engineering, Zhejiang University of Technology, China. His research interests include cutting equipments.

Dapeng Tan, born in 1980, is currently a professor at Zhejiang University of Technology, China. He received his PhD degree from Zhejiang University, China, in 2008. His research interests include mechatronic engineering, ultra precision machining and embedded system technology.

Yongchao Zhang, born in 1981, is currently a lecturer at Taizhou University, China. He received his PhD degree from Shanghai Jiao Tong University, China, in 2012. His research interests include vehicle dynamics and control.

\section{Funding}

Supported by Natural Science Foundation of China (Grant No. 51775501), and Natural Science Foundation of Zhejiang Province, China (Grant Nos. LZ21E050003, LR16E050001, LY17E050004).

\section{Competing interests}

The authors declare no competing financial interests.

\section{Author Details}

${ }^{1}$ College of Mechanical Engineering, Zhejiang University of Technology, Hangzhou 310023, China. ${ }^{2}$ Key Laboratory of Special Purpose Equipment and Advanced Processing Technology, Ministry of Education and Zhejiang Province, Zhejiang University of Technology, Hangzhou 310023, China. ${ }^{3}$ Collaborative Innovation Center of High-end Laser Manufacturing Equipment (National "2011 Plan"), Zhejiang University of Technology, Hangzhou 310023, China. ${ }^{4}$ School of Aeronautical Engineering, Taizhou University, Taizhou 318000, China.

Received: 22 August 2020 Revised: 6 January 2021 Accepted: 29 January 2021

Published online: 10 March 2021

\section{References}

[1] Y B Wang, KJ Hong, J Zou, et al. A CNN-based visual sorting system with cloud-edge computing for flexible manufacturing systems. IEEE Transactions on Industrial Informatics, 2020: 16(7): 4726-4735.

[2] Y Huang, B H Lu, W H Zhao. Application of cloud computing in intelligent machine tool control system. Chinese Journal of Mechanical Engineering, 2018, 54(8): 210-216. (in Chinese)

[3] D M Zhang, J Z Zhang, H W Huang, et al. Machine learning-based prediction of soil compression modulus with application of 1D settlement. Journal of Zhejiang University-Science A, 2020, 21(6): 430-444.

[4] XY Li, J Jie. Ballistic behavior of high strength steel targets during high speed impact of projectile. Journal of Iron and Steel Research, 2020, 32(5): 429-436. (in Chinese) 
[5] L B Zhang, H P LV, D P Tan, et al. An adaptive quantum genetic algorithm for task sequence planning of complex assembly systems. Electronics Letters, 2018, 54(14): 870-871.

[6] Y Z He, L B Zhang, D P Tan, et al. Research on intelligent microgrid monitoring system based on DPWS. Acta Energiae Solaris Sinica, 2015, 36(12): 2979-2985. (in Chinese)

[7] L B Zhang, P H LV, F Xu, et al. Modeling and optimization of CPN collaborative assembling tasks for low-voltage apparatus. Computer Integrated of Manufacturing Systems, 2020, 26(07): 1737-1748. (in chinese)

[8] J K Chen, YY Jin, Z P Zhou. Review of wound roll stress in roll-to-roll manufacturing of flexible electronics. Chinese Science Bulletin, 2019, 64(Z1): 555-565. (in Chinese)

[9] H F Wang, F Zhang, Y F Tong. Digital design and manufacturing technology. Harbin : Harbin Engineering University Press, 2015. (in Chinese)

[10] Z L Cheng, W H Zhou, Z Ding, et al. Estimation of spatiotemporal response of rooted soil using a machine learning approach. Journal of Zhejiang University-Science A, 2020, 21(6): 462-477.

[11] B Zhang, L Gao, Q Xue, et al. Strengths, weaknesses, and applications of computational axial lithography in tissue engineering. Bio-Design and Manufacturing, 2020, 3: 5-6.

[12] L Zhang, Z M Yuan, D P Tan, et al. An improved abrasive flow processing method for complex geometric surfaces of titanium alloy artificial joints. Applied Sciences, 2018, 28(7): 1037.

[13] Y J Rao. Simulation and experimental research on stainless steel sawing process of metal band sawing machine. Hangzhou: Zhejiang University of Technology, 2020. (in Chinese)

[14] Behringer_- The pioneer sawing sawing technology in world. Die \& Mould Manufacture, 2006(2): 62-63. (in Chinese)

[15] Bellinger will launch a new sawing machine in EMO2015. Auto Manufacturing Engineer, 2015(9): 22. (in Chinese)

[16] Horizontal Band Saws - Behringer. https://www.behringersaws.com/. 2020.6.11.

[17] X R Guo. New development trend of feed motion control method of metal band sawing machine. Machine Tool \& Hydraulics, 2016, 44(23): 153-155. (in Chinese)

[18] Amada Machine Tools America Corporation. https://amadamt.com/, 2020.6.11.

[19] ZZ Fang. The design and optimization of the CH360 horizontal CNC band sawing. Hangzhou: Zhejiang University of Technology, 2015. (in (hinese)

[20] Home - KASTO - Saws. Storage. And More. https://www.kasto.com/us/, 2020.6.11.

[21] Machining Products and Technologies|DANOBATGROUP. https://www. danobatgroup.com/en/products, 2020.6.11.

[22] DoALL Sawing Products. https://www.doallsaws.com/, 2020.6.11.

[23] D P Tan, L Li, Y L Zhu, et al. Critical penetration condition and Ekman suction-extraction mechanism of sink vortex. Journal of Zhejiang University-Science A, 2019, 20(1): 61-72.

[24] XX Hu, F Xu, R H Wang. Synchronous sampling-based direct current estimation method for self-sensing active magnetic bearings. Sensors, 2020, 20(12): 3497.

[25] S M Ji, J Q Ge, D P Tan, et al. Wall contact effects of particle-wall collision process in two-phase particle fluid. Journal of Zhejiang UniversityScience A, 2017, 18(12): 958-973.

[26] RFerreira, A Sabbaghi, Q Huang. Automated geometric shape deviation modeling for additive manufacturing systems via Bayesian neural networks. IEEE Transactions on Automation Science and Engineering, 2020, 17(2): 584-598.

[27] Y S Hao, Y S Fan, J Zhang. Service recommendation based on description reconstruction in cloud manufacturing. International Journal of Computer Integrated Manufacturing, 2019, 32(3): 294-306.

[28] J F Lu, T Wang, L Li, et al. Dynamic characteristics and wall effects of bubble bursting in gas-liquid-solid three-phase particle flow. Processes, 2020, 8: 760.

[29] D PTan, PY Li, X H Pan. Application of improved HMM algorithm in slag detection system. Journal of Iron and Steel Research, International, 2009, 16(1): 1-6.

[30] Z M Liu, J Wang. Human-cyber-physical systems: concepts, challenges, and research opportunities. Frontiers of Information Technology \& Electronic Engineering, 2020, 21(11): 1535-1553.
[31] C Li, Q D Xu, J Q Ge, et al. Research on the incidence of soft abrasive flow to a wall and the machining characteristics. Proceeding of Institution of Mechanical Engineers Part B: Journal of Engineering Manufacture, https://doi.org/10.1177/0954405420937559.

[32] Y Guo, N Wang, Z Y Xu, et al. The internet of things-based decision support system for information processing in intelligent manufacturing using data mining technology. Mechanical Systems and Signal Processing, 2020, 142: 106630.

[33] D P Tan, L B Zhang, Q L Ai. An embedded self-adapting network service framework for networked manufacturing system. Journal of Intelligent Manufacturing, 2019, 30(2): 539-556.

[34] C Li, S M Ji, D P Tan. Softness abrasive flow method oriented to tiny scale mold structural surface. International Journal of Advanced Manufacturing Technology, 2012, 61(9-12): 975-987.

[35] S M Ji, XXWeng, D P Tan. Analytical method of softness abrasive twophase flow field based on 2D model of LSM. Acta Physica Sinica, 2012, 61(1): 010205

[36] D P Tan, Y S Ni, L B Zhang. Two-phase sink vortex suction mechanism and penetration dynamic characteristics in ladle teeming process. Journal of Iron and Steel Research, International, 2017, 24(7): 669-677.

[37] D P Tan, L Li, Z C Yin, et al. Ekman boundary layer mass transfer mechanism of free sink vortex. International Journal of Heat and Mass Transfer, 2020, 150: 119250.

[38] Q S Jiang, D P Tan, Y B Li, et al. Object detection and classification of metal polishing shaft surface defects based on convolutional neural network deep learning. Applied Sciences, 2020, 10(1): 87

[39] J Li, S M Ji, D P Tan. Improved soft abrasive flow finishing method based on turbulent kinetic energy enhancing. Chinese Journal of Mechanical Engineering, 2017, 30(2): 301-309.

[40] L Jia, H Y Dai, Y Song. Test technology research and fatigue damage prediction of a car body based on dynamic simulation load spectrum. Journal of Zhejiang University Science A, 2020, 21(11): 923-937.

[41] Q Gao, W Wu, J G Zhi, et al. Effect of a self-rotating oxygen lance system on mass transfer between slag and molten steel. Journal of Iron and Steel Research, International, 2020, https://doi.org/10.1007/s42243-02000515-9.

[42] L Li, D P Tan, TWang, et al. Multiphase coupling mechanism of free surface vortex and the vibration-based sensing method. Energy, 2020, 216: 119136

[43] L Li, H Qi, Z C Yin, et al. Investigation on the multiphase sink vortex Ekman pumping effects by CFD-DEM coupling method. Powder Technology, 2020, 360: 462-480.

[44] J C Zhang, T Zhang, YT Yang. Microstructure and properties evolution of $\mathrm{Nb}$-bearing medium $\mathrm{Cr}$ wear-resistant cast steel during heat treatment. Journal of Iron and Steel Research, International, 2020, https://doi. org/10.1007/s42243-020-00510-0.

[45] C Li, Q D Xu, J Q Ge, et al. Study of soft abrasive flow field measurement based on particle image velocimetry. The International Journal of Advanced Manufacturing Technology, 2020, 109(7-8): 2039-2047.

[46] L Qiu, X Zeng, S M Ji, et al. Research on abrasive belt based machining technology for milling shaped aero-engine blades. Tehnicki Vjesnik Technical Gazette, 2019, 26(5): 1353-1362.

[47] J XWang, B Cao, X Zeng, et al. Detecting difference between process models using edge network. IEEE Access, 2019, 7: 142916-142925.

[48] D P Tan, S M Ji, M S Jin. Intelligent computer-aided instruction modeling and a method to optimize study strategies for parallel robot instruction. IEEE Transactions on Education, 2013, 56(3): 268-273.

[49] ZY Jia, Y Q Wang, F J Wang. Research on measure-redesign-machining integration manufacturing method for complicated surface parts with high performance. Journal of Mechanical Engineering, 2013, 49(19): 126-138. (in Chinese)

[50] X P Guan, CL Chen, C L Chen, et al. Towards the integration of sensing, transmission and control for industrial network systems: Challenges and recent developments. Acta Automatica Sinica, 2019, 45(1): 27-38. (in (hinese)

[51] B Tao, XW Zhao, R P Li. Research on robotic measurement-operationmachining technology and its application. China Mechanical Engineering, 2020, 31 (1): 49-56. (in Chinese)

[52] A Nascimento, R Flesch, C Flesch. Data-driven soft sensor for the estimation of sound power levels of refrigeration compressors through 
vibration measurements. IEEE Transactions on Industrial Electronics, 2020, 67(8): 7065-7072.

[53] N A Fountas, N M Vaxevanidis. Intelligent 3D tool path planning for optimized 3-axis sculptured surface CNC machining through digitized data evaluation and swarm-based evolutionary algorithms. Measurement, 2020, 158: 107678.

[54] B Strbac, B Acko, S Havrlisan, et al. Investigation of the effect of temperature and other significant factors on systematic error and measurement uncertainty in CMM measurements by applying design of experiments. Measurement, 2020, 158: 107692.

[55] K Scicluna, C S Staines, R Raute. Sensorless low/zero speed estimation for permanent magnet synchronous machine using a search-based real-time commissioning method. IEEE Transactions on Industrial Electronics, 2020, 67(8): 6010-6018.

[56] J X Wang, D P Tan, B Cao. An independent path-based process recommendation algorithm for improving biomedical process modeling. Electronics Letters, 2020, 56(11): 531-533.

[57] L Li, J F Lu, H Fang, et al. Lattice Boltzmann method for fluid-thermal systems: status, hotspots, trends and outlook. IEEE Access, 2020, 8 : 27649-27675.

[58] XX Hu, F Xu, D P Tan, et al. A synchronous sampling-based direct current estimation method for self-sensing active magnetic bearings. Journal of Zhejiang University-Science A, 2020, 21(5): 401-405.

[59] L Zhang, J S Wang, D P Tan, et al. Gas compensation based abrasive flow processing method for complex titanium alloy surfaces. International Journal of Advanced Manufacturing Technology, 2017, 92(9-12): 3385-3397.

[60] J Q Ge, S M Ji, D P Tan, et al. A gas-liquid-solid three-phase abrasive flow processing method based on bubble collapsing. International Journal of Advanced Manufacturing Technology, 2018, 95(1-4): 1069-1085.

[61] M G Wu, C D Lu, D P Tan, et al. Effects of metal buffer layer for amorphous carbon film of 304 stainless steel bipolar plate. Thin Solid Films, 2016, 616: 507-514

[62] D P Tan, S M Ji, Y Z Fu. An improved soft abrasive flow finishing method based on fluid collision theory. International Journal of Advanced Manufacturing Technology, 2016, 85(5): 1261-1274.

[63] Y Z Gu, D W Zuo, W M Xu. A modal analysis and optimization of physiotherapy appliance bed structure. Machine Building \& Automation, 2009, 1: 36-39. (in Chinese)

[64] L Jiang, D Xiang, P Mou, et al. Study of gearbox's robust optimization design. Machinery Design \& Manufacture, 2018, S1: 14-16. (in Chinese)

[65] Y H Li, Q Zang, J Zhang. Robust and optimal design of gear box for high speed train. Journal of Dalian Jiaotong University, 2015(6): 29-33. (in (hinese)

[66] JTWang, J G Lu, B XWang, et.al. Application of the food chain conduction response algorithm in the gearbox optimization. Computer Integrated Manufacturing Systems, 2017: 1-18. (in Chinese)

[67] C Liu, Z D Fang, X J Zhang, et al. An efficient modeling method for coupled vibration analysis of double-helical gear reduction. Noise Control Engineering Journal, 2017, 65(2): 121-137.

[68] G Belingardi, V Cuffaro, F Cura. Multibody approach for the dynamic analysis of gears transmission for an electric vehicle. Proceedings of The Institution of Mechanical Engineers Part C - Journal of Mechanical Engineering Science, 2018, 232(1): 57-65.

[69] C Osorio, G Koch, H Pinheiro, et al. Robust current control of grid-tied inverters affected by LCL filter soft-saturation. IEEE Transactions on Industrial Electronics, 2020, 67(8): 6550-6561.

[70] C Li, S M Ji, D P Tan. Multiple-loop digital control method for $400 \mathrm{~Hz}$ inverter system based on phase feedback. IEEE Transactions on Power Electronics, 2013, 28(1): 408-417.

[71] D P Tan, T Yang, J Zhao, et al. Free sink vortex Ekman suction-extraction evolution mechanism. Acta Physica Sinica, 2016, 65(5): 054701.

[72] JW Fan, X F Wang, D J Chen, et al. Error sentivity analysis of machine tools based on mufti-body kinematic theory. Chinese High Technology Letters, 2013, 23(03): 318-324. (in Chinese)

[73] M Ge, S M Ji, D P Tan. Erosion analysis and experimental research of gas-liquid-solid soft abrasive flow polishing based on cavitation effects. International Journal of Advanced Manufacturing Technology, 2020, 9: 40. https://doi.org/10.1016/j.jamt.

[74] YW Hu, H Chen, D D Chen, et al. A flexible hybrid PWM strategy based novel cascaded grid simulator with improved internal model repetitive controller. Frontiers of Information Technology \& Electronic Engineering, 2020, https://doi.org/10.1631/FITEE.1700728.

[75] YYWang, PC Ni, D H Wen, et al. Dynamic performance optimization of circular sawing machine gearbox. Applied Sciences, 2019, 9(20): 4458.

[76] X Zeng, S M Ji, M S Jin, et al. Research on dynamic characteristic of softness consolidation abrasives in machining process. International Journal of Advanced Manufacturing Technology, 2016, 82(5-8): 1115-1125.

[77] J L Chen, F Xu, D P Tan. A control method for agricultural greenhouse heating based on computational fluid dynamics and energy prediction model. Applied Energy, 2015, 141: 106-118.

[78] S H Zheng, Y K Yu, M Z Qiu, et al. A modal analysis of vibration response of a cracked fluid-filled cylindrical shell. Applied Mathematical Modelling, 2021, 91: 934-958

[79] X Zeng, S M Ji, M S Jin, et al. Investigation on machining characteristic of pneumatic wheel based on softness consolidation abrasives. International Journal of Precision Engineering and Manufacturing, 2014, 15(10): 2031-039.

[80] S C Gong, W D Huang, N L Tai, et al. Complex network based comprehensive evaluation method of topology for electro-thermal microgrid. Automation of Electric Power Systems, 2019, 43(23): 173-189. (in Chinese)

[81] Z H Wang, Z H Liu. Partial synchronization over complex networks: chimera states, remote synchronization and group synchronization. Acta Physica Sinica, 2020, 69(8): 59-72. (in Chinese)

[82] D P Tan, L B Zhang. A WP-based nonlinear vibration sensing method for invisible liquid steel slag detection. Sensors and Actuators B, 2014, 202: 1257-1269.

[83] D P Tan, L Li, Y L Zhu, et al. An embedded cloud database service method for distributed industry monitoring. IEEE Transactions on Industrial Informatics, 2018, 14(7): 2881-2893.

[84] EY Chen, Y C Shi, G Y Xu. Evaluation and improvement of UPnP service discovery algorithm. Journal of Tsinghua University(Science and Technology), 2006. 4: 600-608. (in Chinese)

[85] R C Tang, X Feng, X Q Ding, Contest-based content adaptation algorithm and its application in UPnP AV. Journal of Jilin University (Engineering and Technology Edition), 2014, 44(5): 1441-1446. (in Chinese)

[86] CWu, X N Wang, H L Xiao, et al. Design and implement of middleware for high performance computing environment based on message bus. Chinese High Technology Letters, 2020, 30(3): 248-258. (in Chinese)

[87] B FWu. Design of loT middleware based on microservices architecture. Computer Science, 2019, 46(S1): 580-604. (in Chinese)

[88] P C Xiong, Y S Fan. Middleware for consistent data interaction in distributed workflow system. Journal of Tsinghua University (Science and Technology), 2008, 7: 1213-1216. (in Chinese)

[89] D P Tan, PY Li, X H Pan. Computer integrated manufacturing systems. Computer Integrated Manufacturing Systems, 2009, 15(1): 131-134. (in Chinese)

[90] Y Pan, S M Ji, D P Tan, et al. Cavitation based soft abrasive flow processing method. International Journal of Advanced Manufacturing Technology, 2020, 109(9): 2587-2602.

[91] L B Zhang, Y Z He, F Xu, et al. Monitoring method of micro-grid based on DPWS network middleware and Petri nets. Transactions of the Chinese Society of Agricultural Engineering, 2014, 30(20): 163-171. (in Chinese)

[92] $\mathrm{F} \mathrm{Xu,} \mathrm{Y} \mathrm{K} \mathrm{Yang,} \mathrm{D} \mathrm{P} \mathrm{Tan,} \mathrm{et} \mathrm{al.} \mathrm{Monitoring} \mathrm{architecture} \mathrm{of} \mathrm{microgrid} \mathrm{and}$ adaptive service method based on DPWS. Automation of Electric Power Systems, 2015, 39(19): 34-53. (in Chinese)

[93] J F Lu, TWang, L Li, et al. Dynamic characteristics and wall effects of the bubble bursting in gas-liquid-solid three-phase particle flow. Processes, 2020, 8: 760.

[94] D P Tan, PY Li, X H Pan. Intelligent industry monitoring network architecture UPnP based. Chinese Journal of Electronics, 2008, 17(4): 607-610. (in Chinese)

[95] X Wang, S K Ong, A Y C Nee. A comprehensive survey of ubiquitous manufacturing research. International Journal of Production Research, 2019, 30(2): 539-556.

[96] China Mobile. China Mobile officially released 5 G module middleware and solutions. http://m.elecfans.com/article/1167135.html, 2020. (in Chinese)

[97] L Zheng, PY Jiang, L H Qiao, et al. Challenges and frontiers of manufacturing systems. Journal of Mechanical Engineering, 2010, 46(21): 124-136. (in Chinese) 
[98] HQWu. New features of network society and industry challenges in the $5 \mathrm{G}$ era. Journal of Chongqing University of Posts and Telecommunications (Natural Science Edition), 2020, 32(2): 171-176. (in Chinese)

[99] D P Tan, P Y Li, Y X Ji, et al. SA-ANN-based slag carry-over detection method and the embedded WME platform. IEEE Transactions on Industrial Electronics, 2013, 60(10): 4702-4713.

[100] S M Ji, F Q Xiao, D P Tan. Analytical method for softness abrasive flow field based on discrete phase model. Science China-Technological Sciences, 2010, 53(10): 2867-2877.

[101] D P Tan, S M Ji, P Y Li, et al. Development of vibration style ladle slag detection method and the key technologies. Science China-Technological Sciences, 2010, 53(9): 2378-2387.

[102] ST Chen, D P Tan. A SA-ANN-based modeling method for human cognition mechanism and the PSACO cognition algorithm. Complexity, 2018, 2018: 6264124.

[103] B H Li, X D Cai, B C Hou, et al. Computer integrated manufacturing systems. Computer Integrated Manufacturing Systems, 2019, 25(12): 2997-3012. (in Chinese)

[104] Z D Shan, Y Zhou, Y Z Li. Process optimization and decision-making model of service-oriented manufacturing network based on cloud computer. Computer Integrated Manufacturing Systems, 2019, 25(12): 3139-3148. (in Chinese)

[105] J F Man, L Q Zhao, C Peng, et al. Computer Integrated Manufacturing Systems. Computer Integrated Manufacturing Systems, 2020, DOl: 11.5946.tp.20200502.0852.002. (in Chinese)

[106] P Helo, D Phuong, Y Q G Hao. Cloud manufacturing - scheduling as a service for sheet metal manufacturing. Computers \& Operations Research, 2019, 110: 208-219.

[107] A Simeone, B Deng, A Caggiano. Resource efficiency enhancement in sheet metal cutting industrial networks through cloud manufacturing International Journal of Advanced Manufacturing Technology, 2020, 61: 101832.20: 107(3-4): 1345-1365.

[108] M H Mourad, A Nassehi, D Schaefer, et al. Assessment of interoperability in cloud manufacturing. Robotics and Computer - Integrated Manufacturing, 2020, 61: 101832

[109] E Aghamohammadzadeh, O F Valilai. A novel cloud manufacturing service composition platform enabled by Blockchain technology.
International Journal of Production Research, 2020, https://doi. org/10.1080/00207543.2020.1715507.

[110] W Guo, K Q Zhang, Z L Cui, et al. A cloud resources placement method supporting SaaS applications with multi-dimensional and heterogen requirements. Chinese Journal of Computers, 2018, 41(6): 1005-1017. (in Chinese)

[111] D P Tan, ST Chen, G J Bao, et al. An embedded lightweight GUI component library and the ergonomics optimization method for industry process monitoring. Frontiers of Information Technology \& Electronic Engineering, 2018, 19(5): 604-625.

[112] H B Li, F Cheng, Juan A Robledo-Lara, et al. Fabrication of paper-based devices for in vitro tissue modeling. Bio-Design and Manufacturing, 2020, 3: 252-265.

[113] Z C Yin, J F Lu, L Li, et al. Optimized scheme for accelerating the slagging reaction and slag-metal-gas emulsification in a basic oxygen furnace. Applied Sciences, 2020, 10(15): 5101

[114] CXWang, Z Peng, Y Z Wang, et al. Research on structure designation and cutting performance of the damping tool. Cemented Carbide, 2018, 35(06): 441-446. (in Chinese)

[115] Y Q Yang, Y W Pian, Y F Wang. Experimental tests of damped milling cutter based on embedded passive damper. Journal of Vibration, Measurement \& Diagnosis, 2019, 39(06): 1177-1182+1357. (in Chinese)

[116] S Ema, E Marui. Suppression of chatter vibration of boring tools using impact dampers. International Journal of Machine Tools and Manufacture, 2000, 40(8): 1141-1156.

[117] S K Choudhury, N N Goudimenko, V A Kudinov. On-line control of machine tool vibration in turning. International Journal of Machine Tools \&Manufacture, 1997, 37(6): 801-811.

[118] C H Chiou, M S Hong, K F Ehmann. The feasibility of eigenstructure assignment for machining chatter control. International Journal of Machine Tools and Manufacture, 2003, 43(15): 1603-1620.

[119] TR Kong. Study on the theory and method for MR intelligent boring bar with self-chatter-suppression. Hangzhou: Zhejiang University, 2009. (in Chinese)

\section{Submit your manuscript to a SpringerOpen ${ }^{\circ}$ journal and benefit from:}

- Convenient online submission

- Rigorous peer review

- Open access: articles freely available online

- High visibility within the field

- Retaining the copyright to your article

Submit your next manuscript at springeropen.com 